\title{
EXACT SOLUTIONS FOR SPECTRA AND GREEN'S FUNCTIONS IN RANDOM ONE-DIMENSIONAL SYSTEMS
}

\author{
Th.M. NIEUWENHUIZEN \\ Institute for Theoretical Physics, Princetonplein 5, P.O. Box 80.006, 3508 TA Utrecht, \\ The Netherlands
}

Received 22 August 1983

For chains of harmonic oscillators with random masses a set of equations is derived, which determine the spatial Fourier components of the average one-particle Green's function. These equations are valid for complex values of the frequency. A relation between the spectral density and functions introduced by Schmidt is discussed. Exact solutions for this Green's function and the less complicated characteristic function - the analytic continuation into the complex frequency plane of the accumulated spectral density and the inverse localization length of the eigenfunctions - are derived for exponential distributions of the masses. For some cases the characteristic function is calculated numerically. For gamma distributions the equations are cast in the form of ordinary, higher order differential equations; these have been solved numerically for determining the characteristic function. For arbitrary mass distributions a cumulant expansion and a peculiar symmetry of the Green's function are discussed.

The method is also applied to chains where the spring constants and/or the masses have random values. Also for these systems exact solutions are discussed; for exponential distributions, e.g., of both masses and spring constants the characteristic function is expressed in Bessel functions. The relation with certain random relaxation models is shown. Finally, $X-Y$ Hamiltonians with random exchange constants and/or magnetic fields - or, equivalently, tight-binding electron models with diagonal and/or off-diagonal disorder - are considered. Here the Green's function does not depend on the wave number if the distribution of exchange constants is symmetric around the origin. New solutions for the characteristic function and Green's function are derived for a number of cases, including exponentially distributed magnetic fields and power law distributed exchange constants.

\section{Introduction}

In ordered as well as in random systems - which will be the subject of this paper-thermodynamic functions are the first quantities of interest. In onedimensional systems where the equation of motion - which may be a classical one or a Schrödinger or a Master equation - is linear they are determined by the spectral density of eigenfrequencies or the density of states. The characteristic function is the indefinite integral of the trace of the one-particle Green's function; it extends the accumulated spectral density into the complex frequency plane. The real part of this function along its cut is connected to the exponential localization of the eigenfunctions ${ }^{1,2}$ ); its imaginary part is proportional to the accumulated density of states. Exact solutions for either the density of states or the character- 
istic function have been found for random harmonic chains by Dyson ${ }^{3}$ ) and Domb et al. ${ }^{4}$ ), for a particle in a Gaussian white-noise potential by Frisch and Lloyd ${ }^{5}$ ), for tight-binding electron models by Lloyd ${ }^{6}$ ) and for several random Kronig-Penney models by the author ${ }^{7}$ ). In these cases the thermodynamics is known completely. An explicit calculation of the derivative of the specific heat with respect to temperature for the case of a chain consisting of harmonic oscillators for arbitrary distributions of the random masses was reported recently ${ }^{8}$ ).

Time-dependent properties are determined by one-particle Green's functions; they express the solutions of the equations of motion at time $t$ into the initial condition as $u_{k}(t)=\Sigma_{l} G(k, t \mid l, 0) u_{l}(0)$. In random systems the process of averaging over the random variables has to be performed at the right moment; in particular the average of a two-particle Green's function is not equal to the average of the one-particle Green's function squared. Depending on the model one considers the quantities of interest are linear or quadratic in the Green's functions:

i) in disordered harmonic systems $u_{k}(t)$ is the displacement of the $k$ th particle from its equilibrium position; diffusion of displacement and momentum along the chain as well as one-phonon contribution to the differential cross-section for neutron scattering are described by equations that are linear in $u_{k}(t)$ (see also scction 2); diffusion of energy by quadratic ones;

ii) in the case of random walks on (random) lattices $u_{k}(t) \equiv P_{k}(t)$ is the probability for a particle to be at site $k$ at time $t$. Quantities of interest (e.g. frequency dependent hopping conductivity, long time tails in correlation functions) are determined by the one-particle Green's function;

iii) in quantum systems $u_{n}(t)$, or $u(x, t)$, is a probability amplitude and observables are determined by $\left|u_{n}\right|^{2}$. The average one-particle Green's function, however, still determines the density of states at given values of energy and wavenumbers.

In this paper we will calculate the Fourier-Laplace transform (with respect to space and time, respectively) of the average one-particle Green's function. Exact solutions for this function were given by Lloyd ${ }^{6}$ ) for tight binding electron models for arbitrary lattices in arbitrary dimension, provided that the site energies have Cauchy distributions. These distributions, however, have unphysical properties; for instance they yield a divergent zero-point energy per particle ${ }^{2,7}$ ). In order to construct a method that is valid for arbitrary distributions, we restrict ourselves to the case of one space-dimension, although it seems possible to generalize the method to higher dimensions. For obtaining exact solutions, as we will do too, this restriction is unavoidable. Our work extends both a scheme of equations for determining the imaginary part of the Green's function, derived by Halperin ${ }^{9}$ ), into the complex frequency (energy-) plane and a method for obtaining the characteristic function ${ }^{2}$ ).

The set up of the paper is as follows: in section 2.1 for the case of harmonic 
oscillators with random masses, after discussing some applications of the average one-particle Green's function, a straightforward derivation of a scheme of equations is given. The solutions of these equations determine the Fourier-Laplace transform of this Green's function and the characteristic function. In section 2.2 a relation for the spectral density is given, from which we derive easily its boundedness and continuity, provided the mass distribution is absolutely continuous. In section 3.1 we derive explicit solutions for this Green's function and for the characteristic function for the case of exponential mass distributions; in section 3.2 the application to gamma distributions is considered. In section 3.3 it is shown how a straightforward expansion in cumulants of the mass distribution can be obtained.

In section 4.1 we briefly extend the method to harmonic chains where both the masses and springs are random variables; in some specific cases the characteristic function can be expressed in known (Bessel-) functions. In section 4.2 we discuss the relation with the problem of random walks on certain random lattices.

In section 4.3 the relation with $X-Y$ and tight binding electron models is discussed. A summary of the main results is given in section 5 .

\section{Equations for the phonon Green's functions}

\subsection{Random masses}

In this section we discuss a scheme for the evaluation of the Fourier components of the average one-particle Green's function. It is an extension into the complex frequency plane of a method developed by Halperin ${ }^{9}$ ) and also a generalization of a method introduced before ${ }^{2}$ ) for the calculation of the characteristic function. For the sake of clearness the corresponding equations of the last paper will be rederived. We consider an ensemble of chains of harmonic oscillators, connected to their neighbours with ideal springs, with spring constants normalized to unity. The mass of each particle is a random variable, which is independent of the other masses. All masses have the same distribution. The equation for $u_{k}$, the Fourier component with frequency $\omega$ of the displacement of the $k$ th particle from its equilibrium position, has the form

$$
\rho_{k} u_{k}(\xi)-u_{k+1}(\xi)-u_{k-1}(\xi)=0,
$$

where, with $\xi \equiv-\omega^{2}$,

$$
\rho_{k}=2-m_{k} \omega^{2}=2+m_{k} \xi .
$$

We choose fixed boundary conditions

$$
u_{0}=0, u_{1}=1 \text {; }
$$


the requirement that $\boldsymbol{u}_{N+1}(\xi)=0$ fixes the eigenfrequencies $\omega_{1}^{(N)}, \ldots, \omega_{N}^{(N)}$. This equation can be written in the matrix form

$$
(M \xi+\Phi) u=0,
$$

where $M$ is the diagonal mass matrix $\left(M_{k k}=m_{k} ; k=1, \ldots, N\right)$; $\Phi$ is the symmetric force matrix $\left(\Phi_{k k}=2, k=1, \ldots, N ; \Phi_{k, k+1}=\Phi_{k+1, k}=-1, k=1, \ldots, N-1\right.$; else $\left.\Phi_{i j}=0\right)$ and $u$ is a vector with components $u_{k}$. The Green function of this problem is defined as

$$
\mathscr{G}=\frac{1}{M \xi+\Phi}
$$

we will consider the slightly more general Green function

$$
G=\Lambda \mathscr{G} \Lambda^{\dagger}=\Lambda \frac{1}{M \zeta+\Phi} \Lambda^{\dagger},
$$

where $\Lambda$ is a diagonal matrix with elements $\Lambda_{k k}=\lambda_{k}$, which may depend on the type of atom at site $k$. For large systems the average of $G$ will be translationally invariant, since the distribution of the random values of the masses is the same for each site in the chain and independent of the values of the other masses. Therefore $\langle G\rangle$, i.c. the average of $G$ over the masses, is diagonal in Fourier-space ( $q$-space), with components given for finite $N$ by

$$
\begin{aligned}
G^{(N)}(q, \xi) & \equiv\left\langle G_{q q}^{(N)}(\xi)\right\rangle=\frac{1}{N} \sum_{k, l=1}^{N} \mathrm{e}^{-\mathrm{i} q(k-l)}\left\langle G_{k l}^{(N)}(\xi)\right\rangle \\
& =\frac{1}{N} \sum_{i=1}^{N} \sum_{k, l=1}^{N}\left\langle\frac{1}{\xi+\omega_{i}^{(N)^{2}}} \lambda_{k} e_{k}(i) \mathrm{e}^{-\mathrm{i} q k} \lambda_{l}^{*} e_{l}(i) \mathrm{e}^{\mathrm{i} q l}\right\rangle,
\end{aligned}
$$

where, with $\xi_{i}^{(N)}=-\omega_{i}^{(N)^{2}}$,

$$
e_{k}(i)=u_{k}\left(\xi_{i}^{(N)}\left\{\sum_{l=1}^{N} m_{l} u_{l}^{2}\left(\xi_{i}^{(N)}\right)\right\}^{-1 / 2}\right.
$$

The last equality in (2.5a) follows from expansion in eigenfunctions; it will also be discussed later. In a number of fields this Green's function plays an important role. We mention only two applications:

i) contribution of ions to the dielectric constant. In the long wave length approximation the equation of motions of ions with charge $Q_{k}$ in the presence of a local electric field $E_{k} \cos \omega_{0} t$ is given by

$$
m_{k} \ddot{u}_{k}(t)=u_{k+1}(t)+u_{k-1}(t)-2 u_{k}(t)+Q_{k} E_{k} \cos \omega_{0} t .
$$

This equation has an advanced and retarded solution, of which only the last one 
is relevant. For the average dipole moment per particle we obtain

$$
\langle P(t)\rangle=\frac{1}{N} \sum_{k=1}^{N}\left\langle Q_{k} u_{k}(t)\right\rangle=\frac{1}{N} \sum_{k, l=1}^{N} \operatorname{Re}\left\langle Q_{k} \mathscr{G}_{k l}\left(-\omega_{0}^{2}+\mathrm{i} 0\right) Q_{l} E_{l} \mathrm{e}^{\mathrm{i} \omega_{0} t}\right\rangle,
$$

where choosing $+\mathrm{i} 0$ in $\mathscr{G}$ yields the retarded solution. After assuming that the local electric field $E_{l}$ does not depend on $l$ (which needs not be a good approximation in disordered systems), we observe that the r.h.s. of this equation has the form (2.4b), (2.5a) with $\lambda_{k}=Q_{k}$ and $q=0$ (long wave length approximation). Note that the average dipole moment is delayed in time with respect to the local electric field. This does not happen in ordered systems, where the $q=0$ modes can only occur at a finite number - determined by the number of atoms per unit cell and the dimension - of frequencies (resonances). In disordered crystals these resonances are smeared out; the divergence of $\langle P(t)\rangle$ at these frequencies is suppressed, but there occurs a delay. For the calculation of the ionic contribution to the dielectric constant one further needs to know the dependence of the local electric field on the average electric field in the medium and on the polarization. For the case that a large number of non-interacting chains are embedded somehow in a three dimensional crystal, this will be determined by the actual positions of the chains. An example of such a system is $\mathrm{Hg}_{3-\delta} \mathrm{AsF}_{6}{ }^{10}$ ), where, however, only one type of atoms (mercury atoms) can move in onedimensional channels;

ii) for neutron scattering on a one-dimensional system the one-phonon inelastic contribution to the differential cross section per unit solid angle, per unit energy is given by ${ }^{11}$ ) (with $\hbar=1$ )

$$
\frac{\mathrm{d}^{2} \sigma}{\mathrm{d} \Omega \mathrm{d} \omega}=\frac{\left|\boldsymbol{k}^{\prime}\right|}{2 \pi|\boldsymbol{k}|} q^{2} D(q, \omega) .
$$

Here $\boldsymbol{k}\left(\boldsymbol{k}^{\prime}\right)$ is the wave vector of the incident (reflected) neutron, $q$ is the component of $\boldsymbol{k}-\boldsymbol{k}^{\prime}$ along the chain and $\omega$ is the energy transfer from a neutron to the system (creation of a phonon if $\omega>0$, absorption if $\omega<0$ ). The function $D(q, \omega)$ is the Fourier transform of a correlation function

$$
D(q, \omega)=\left\langle\frac{1}{N} \sum_{k, l} b_{k} b_{l} \int_{-\infty}^{\infty} \mathrm{e}^{\mathrm{i} \omega t}\left\langle\left\langle u_{l}(t) u_{k}(0)\right\rangle\right\rangle \mathrm{e}^{-\mathrm{i} q(k-l)}\right\rangle,
$$

where the double angular brackets stand for averaging the quantum mechanical expectation values of the operators with respect to the thermal distribution of the phonon occupation numbers and the single angular brackets stand for averaging with respect to the values of $m_{k}$ (mass of $k$ th atom) and $b_{k}$ (scattering length of $k$ th atom in a crystal). We have also assumed that the equilibrium positions of the atoms are equally spaced, with spacing equal to unity. With methods known 
in ordered systems, see for instance ref. 11, the thermal averages can be carried out and $D$ takes the form

$$
D(q, \omega)=\left[\operatorname{coth} \frac{\omega}{2 k T}+\operatorname{sg}(\omega)\right] \operatorname{Im} G\left(q,-\omega^{2}-\mathrm{i} 0\right),
$$

where the last factor was introduced in (2.4), (2.5) if we identify $\lambda_{k}$ with the scattering length $b_{k}$. Since here only the imaginary part of $G\left(q,-\omega^{2}-\mathrm{i} 0\right)$ is needed $D(q, \omega)$ can be determined from the work of Halperin $\left.{ }^{9}\right)$.

We now proceed with the evaluation of $G(q, \xi)$. The Green's function $\mathscr{G}_{k l}^{(\mathcal{N})}$ satisfies the equation

$$
\rho_{k} \mathscr{G}_{k l}^{(N)}-\mathscr{G}_{k+1, l}^{(N)}-\mathscr{G}_{k-1, l}^{(N)}=\delta_{k, l}
$$

The solution is

$$
\mathscr{G}_{k l}^{(N)}= \begin{cases}\frac{D_{k+1, N} D_{1, l-1}}{D_{1, N}}, & k \geqslant l, \\ \frac{D_{l+1, N} D_{1, k-1}}{D_{1, N}}, & k \leqslant l,\end{cases}
$$

where, together with $D_{k, k-1}=1$ and $D_{k, k-2}=0$,

$$
D_{k, l}=\left|\begin{array}{ccccc}
\rho_{k} & -1 & & \emptyset \\
-1 & \rho_{k+1} & -1 & \\
& -1 & \cdot & & \\
\emptyset & & & . & -1 \\
\emptyset & & & -1 & \rho_{l}
\end{array}\right|,
$$

see for instance Herbert and Jones ${ }^{12}$.

Note that the solution of (2.1) with fixed boundary conditions $u_{0}=0, u_{1}=1$ is given by $u_{k}=D_{1, k-1}$. Using the equality

$$
D_{k+1, N} D_{1, N-1}-D_{k+1, N-1} D_{1, N}=D_{1, k-1},
$$

which can be proven by induction, we find

$$
\mathscr{H}_{k i}^{(N)} \equiv \mathscr{G}_{k l}^{(N)}-\mathscr{G}_{k l}^{(N-1)}=\frac{D_{1, l-1} D_{1, k-1}}{D_{1, N-1} D_{1, N}}=\frac{u_{k} u_{l}}{u_{N} u_{N+1}} .
$$

In a similar way we define $H_{k l}^{(N)}$ in terms of $G_{k l}^{(N)}$; its Fourier transform is given by

$$
H_{q q}^{(N)}(\zeta)=\sum_{k, l=1}^{N} H_{k l}^{(N)} \mathrm{e}^{-\mathrm{i}(k-1) q}=\sum_{k, l=1}^{N} \frac{\lambda_{k} u_{k} \mathrm{e}^{-\mathrm{i} q k} \lambda_{l}^{*} u_{l} \mathrm{e}^{\mathrm{i} q l}}{u_{N} u_{N+1}} .
$$

Here the $u_{k}$ depend parametrically on $\xi$. A more familiar form is obtained if we 
use the equality

$$
u_{N}(\xi) u_{N+1}^{\prime}(\xi)-u_{N+1}(\xi) u_{N}^{\prime}(\xi)=\sum_{j=1}^{N} m_{j} u_{j}^{2}(\xi)
$$

for $\xi$ close to one of the eigenvalues $\xi_{i}^{(N)}=-\omega_{i}^{(N)^{2}}$, where, by definition, $u_{N+1}(\xi)=0$. We then obtain, together with $G_{q q}^{(0)}=0$,

$$
H_{q q}^{(N)}(\xi)=N G_{q q}^{(N)}(\xi)-(N-1) G_{q q}^{(N-1)}(\xi),
$$

where $G_{q q}^{(M)}$ was defined in (2.5). The equality between (2.15) and (2.17) holds, because both expressions are equal to the quotient of polynomials of degree $2 N-2$ and $2 N-1$, respectively, and have the same poles and equal residues. From this equation we can solve the desired quantity $G_{q q}^{(N)}$ :

$$
G_{q q}^{(N)}(\xi)=\frac{1}{N} \sum_{n=1}^{N} \dot{H}_{q q}^{(n)}
$$

In the following it will become clear that the $H_{q q}^{(n)}$ have a limit as $n \rightarrow \infty$. Taking the average over all values of the masses $m_{k}$ and parameters $\lambda_{k}$ we obtain for the average Green's function in the limit $N \rightarrow \infty$

$$
G(q, \xi)=\left\langle G_{q q}(\xi)\right\rangle=\left\langle H_{q q}\right\rangle .
$$

For the evaluation of the r.h.s. of this equation we introduce the quantities

$$
\begin{aligned}
& z_{k}(\xi)=\frac{u_{k}(\xi)}{u_{k+1}(\xi)} \\
& U_{k}(q, \xi)=\frac{\mathrm{e}^{\mathrm{i} q(k+1)}}{u_{k+1}(\xi)} \sum_{j=1}^{k} \lambda_{j} \mathrm{e}^{-\mathrm{i} q j} u_{j}(\xi) \\
& \bar{U}_{k}(q, \xi)=\frac{\mathrm{e}^{-\mathrm{i} q(k+1)}}{u_{k+1}(\xi)} \sum_{j=1}^{k} \lambda_{j}^{*} \mathrm{e}^{\mathrm{i} q j} u_{j}(\xi) .
\end{aligned}
$$

The quantity $U_{k}$ was also used by Halperin ${ }^{9}$ ). Note that we do not define $U_{k}$ as the complex conjugate of $U_{k}$, since then we would loose the analytic properties in the complex $\xi$-plane. For fixed values of $q$ and $\xi$ these quantities satisfy the equations, as follows from (2.1),

$$
\begin{aligned}
& z_{k}=\frac{1}{\rho_{k}-z_{k-1}}, \quad z_{0}=0, \\
& U_{k}=z_{k} \mathrm{e}^{\mathrm{i} q}\left(U_{k-1}+\lambda_{k}\right), \quad U_{0}=0, \\
& \bar{U}_{k}=z_{k} \mathrm{e}^{-\mathrm{i} q}\left(\bar{U}_{k-1}+\lambda_{k}^{*}\right), \quad \bar{U}_{0}=0 .
\end{aligned}
$$

The $z_{k}$ are analytic functions of $\xi$, with single poles on the negative real $\xi$-axis; one has $\operatorname{Im} z_{k}(\xi) \lessgtr 0$ if $\operatorname{Im} \xi \gtrless 0$. Because the $u_{k}(\xi)$ are polynomials in $\xi$, with only 
zeros for negative real $\xi$, we can introduce the functions, analytic in $u$ and $\xi$,

$$
\begin{aligned}
& D^{(N)}(u ; \xi)=\left\langle\log \left(\frac{1}{z_{N}(\xi)}-\frac{1}{u}\right)\right\rangle, \\
& R_{0}^{(N)}(u ; \xi)=\left\langle\frac{1}{u-z_{N}(\xi)}\right\rangle, \\
& R_{1}^{(N)}(u ; q ; \xi)=\left\langle\frac{U_{N}(q, \xi)}{u-z_{N}(\xi)}\right\rangle ; \\
& \bar{R}_{1}^{(N)}(u ; q ; \xi)=\left\langle\frac{\bar{U}_{N}(q ; \xi)}{u-z_{N}(\xi)}\right\rangle \\
& R_{2}^{(N)}(u ; q, \xi)=\left\langle\frac{U_{N}(q ; \xi) \bar{U}_{N}(q ; \xi)}{u-z_{N}(\xi)}\right\rangle .
\end{aligned}
$$

They have no singularities in the combined parts of the complex $u$ and $\xi$ plane, where $\operatorname{sg}(\operatorname{Im} u)=\operatorname{sg}(\operatorname{Im} \xi)$. The function $D$ was introduced before $\left.{ }^{2}\right)$ for the calculation of the characteristic function

$$
\Omega(\xi)=\int \log \left(\xi+\omega^{2}\right) \mathrm{d} H\left(\omega^{2}\right)+\langle\log m\rangle,
$$

where $H$ is the average accumulated spectral density. Along the negative real $\xi$-axis this function has the decomposition

$$
\Omega\left(-\omega^{2} \pm \mathrm{i} 0\right)=\gamma\left(\omega^{2}\right) \pm \mathrm{i} \pi H\left(\omega^{2}\right) .
$$

The quantity $\gamma\left(\omega^{2}\right)$ is often called "exponential growth rate"13) or "inverse localization length"1); it is positive for disordered chains, which is connected to the exponential localization of all the eigenfunctions ${ }^{1,13,14}$ ). From the definition (2.4a) it can be shown that for $N \rightarrow \infty$

$$
\Omega^{\prime}(\xi)=\left\langle\frac{1}{N} \operatorname{Tr} \frac{1}{\xi+M^{-1} \Phi}\right\rangle=\int_{-\pi}^{\pi} \frac{\mathrm{d} q}{2 \pi \xi}(1-2(1-\cos q) \mathscr{G}(q, \xi)) .
$$

Comparing this with (2.24) we see that the quantity $\rho\left(\omega^{2}, q\right)=\left(2(1-\cos q) / \pi \omega^{2}\right)$ $\operatorname{Im} \mathscr{G}\left(q,-\omega^{2}-\mathrm{i} 0\right)$ may be interpreted as the density of states at given wave number $q$. The total density of states then follows as $\mathrm{d} H / \mathrm{d} \omega^{2}=\int_{-\pi}^{\pi}(\mathrm{d} q / 2 \pi) \rho\left(\omega^{2}, q\right)$.

Of course the function $R_{0}(u)$ is equal to $D^{\prime}(u)+1 / u$; we only introduce it separately for convenience. As $N \rightarrow \infty$ we obtain from (2.21) a closed set of equations

$$
D(u)=\left\langle D\left(\rho_{j}-\frac{1}{u}\right)+\log \left(\rho_{j}-\frac{1}{u}\right)\right\rangle-D(\infty), \quad\left(\rho_{j}=2+m_{j} \xi\right),
$$




$$
\begin{aligned}
R_{0}(u)= & \frac{1}{u^{2}}\left\langle R_{0}\left(\rho_{j}-\frac{1}{u}\right)\right\rangle+\frac{1}{u}, \\
R_{1}(u)= & \frac{\mathrm{e}^{\mathrm{i} q}}{u}\left\langle R_{1}\left(\rho_{j}-\frac{1}{u}\right)+\lambda_{j} R_{0}\left(\rho_{j}-\frac{1}{u}\right)\right\rangle, \\
\bar{R}_{1}(u)= & \frac{\mathrm{e}^{-\mathrm{i} q}}{u}\left\langle\bar{R}_{1}\left(\rho_{j}-\frac{1}{u}\right)+\lambda_{j}^{*} R_{0}\left(\rho_{j}-\frac{1}{u}\right)\right\rangle, \\
R_{2}(u)= & \left\langle R_{2}\left(\rho_{j}-\frac{1}{u}\right)+\lambda_{j}^{*} R_{1}\left(\rho_{j}-\frac{1}{u}\right)+\lambda_{j} \bar{R}_{1}\left(\rho_{j}-\frac{1}{u}\right)\right. \\
& \left.+\lambda_{j}^{*} \lambda_{j} R_{0}\left(\rho_{j}-\frac{1}{u}\right)\right\rangle+R_{2}(0) .
\end{aligned}
$$

Here the angular brackets indicate averaging with respect to $m_{j}$ and $\lambda_{j}$ and we suppresssed the indices $q$ and $\xi$. In ${ }^{2}$ ) it was shown that the characteristic function is given by

$$
\Omega(\xi)=D(\infty ; \xi),
$$

while from (2.15), (2.19), (2.20 and (2.23) it follows that the Green's function is equal to

$$
G(q, \xi)=-R_{2}(0 ; q ; \xi) .
$$

Eqs. (2.26) (2.28) together constitute a scheme for the evaluation of the characteristic function and the average one-particle Green's function for real or complex values of $\xi$. They are valid for arbitrary distributions of the masses (in particular for all cases discussed by Alexander et al. $\left.{ }^{15}\right)$ ) and the parameters $\lambda_{j}$.

The solutions to these equations are unique as can be shown using arguments of analyticity ${ }^{2}$ ). Similar equations are valid for other models, as will be discussed in subsequent sections. If $\lambda_{j}=1$ for special distributions of the masses explicit solutions can be constructed (sections 3.1, 3.2); for arbitrary distributions expansions in cumulants can be made (section 3.3). It should also be possible to deduce results obtained by Alexander et al. ${ }^{15}$ ) and Stephen and Kariotis ${ }^{16}$ ) for the frequency dependent diffusion coefficient. In a certain model this quantity is proportional to $\mathscr{G}(0, \xi)$ (section 4.2 ).

Here we still discuss the relation with the functions $P_{0}, P_{1}$ and $P_{2}$, introduced by Halperin $\left.{ }^{9}\right)$, for the calculation of the wave vector dependent spectral density $A(q, \omega) \equiv 2|\omega| / \pi \operatorname{Im} G\left(q,-\omega^{2}-\mathrm{i} 0\right)$ for real values of $\omega$ (negative $\xi=-\omega^{2}$ ). These functions are defined by

$$
\begin{aligned}
& P_{0}(u ; N)=\left\langle\delta\left(u-z_{N}\right)\right\rangle, \\
& P_{1}(u ; N)=\left\langle U_{N} \delta\left(u-z_{N}\right)\right\rangle, \\
& P_{2}(u ; N)=\left\langle\left|U_{N}\right|^{2} \delta\left(u-z_{N}\right)\right\rangle .
\end{aligned}
$$


Comparing with (2.22) we see that $P_{0}(u ; N)=(1 / \pi) \operatorname{Im} R_{0}^{(N)}(u-\mathrm{i} 0)$ and a similar relation exists between $P_{1}$ and $R_{1}^{(N)}$. The relation between $P_{2}$ and $R_{2}$, however, is given by

$$
P_{2}(u ; N)-P_{2}(\infty ; N)=\frac{1}{\pi} \operatorname{Im} R_{2}^{(N)}(u-\mathrm{i} 0) .
$$

This is caused by the fact that $R_{2}^{(N)}(u ; q ; \xi)$ does not only have singularities at the points where $u=z_{N}\left(-\omega^{2}\right)$, but also at the points where $u_{N+1}\left(-\omega^{2}\right)=0$, i.e. $z_{N}\left(-\omega^{2}\right)=\infty$. Therefore, unlike $R_{2}^{(N)}(u), P_{2}(u ; N)$ does not vanish as $u \rightarrow \infty$. With the above mentioned relations the equations for the functions $P_{0}, P_{1}$ and $P_{2}$, derived by Halperin ${ }^{9}$ ), follow also from (2.26). Finally, the identity $A(q, \omega)=2|\omega|$ $\lim _{N \rightarrow \infty}(1 / N) P_{2}(u ; N)$ for fixed value of $u$ follows because from $(2.28),(2.30)$ we have in the limit $N \rightarrow \infty$

$$
\begin{aligned}
A(q, \omega) & =-\frac{2|\omega|}{\pi} \operatorname{Im} R_{2}^{(N)}(0-\mathrm{i} 0)=2|\omega|\left\{P_{2}(\infty ; N)-P_{2}(0 ; N)\right\} \\
& =2|\omega|\left\{P_{2}(\infty ; N)-P_{2}(\infty ; N-1)\right\} .
\end{aligned}
$$

The last equality follows, for instance, from the definition of $P_{2}$, eq. (2.29).

\subsection{On the existence of the spectral density}

In this section we derive a formula for $\mathrm{d} \Omega / \mathrm{d} \xi$ in terms of the function $R_{0}(u ; \xi)$ and an expression for $G(q, \xi)$ in terms of $R_{0}, R_{1}$ and $\bar{R}_{1}$. The fact that the function $R_{2}$ is not needed may be of practical use; these equations can also be used to investigate the existence of the spectral density in a simple manner.

We start with the derivative of eq. (2.26a) with respect to $\xi$ in the point $1 / u$ and define $E(u)=D(u)-D(\infty)+\log (-u)$;

$$
\partial_{\xi} E\left(\frac{1}{u}\right)=\left\langle\partial_{\xi} E\left(\rho_{j}-u\right)+m_{j} R_{0}\left(\rho_{j}-u\right)\right\rangle-\partial_{\xi} E(0) .
$$

In this equation we regard $R_{0}$ known as solution of (2.26b). The unknown terms $\partial_{\xi} E$ are found to cancel if we multiply both sides by $R_{0}(u)$ and integrate over $u$ from $-A$ to $+A$ for $A \rightarrow \infty$. If we use the definition (2.22) for $R_{0}(u)$, interchange the integral over $u$ and the averaging over the values of the masses, use the fact that $\operatorname{sg}\left(\operatorname{Im} z_{j}\right)=-\operatorname{sg}(\operatorname{Im} \xi)$ and the equation of motion $(2.26 \mathrm{~b})$ for $R_{0}(u)$, we can prove the properties

i) $\lim _{A \rightarrow \infty} \int_{-A}^{A} R_{0}(u) \mathrm{d} u=-\pi \mathrm{i} \operatorname{sg}(\operatorname{Im} \xi)$, 
ii) $\lim _{A \rightarrow \infty} \int_{-A}^{A} R_{0}(u)\left\{\partial_{\xi} E\left(\frac{1}{u}\right)-\partial_{\xi}\left\langle E\left(\rho_{j}-u\right)\right\rangle\right\}=\pi \mathrm{i} \operatorname{sg}(\operatorname{Im} \xi) \partial_{\xi} E(0)$ $=-\pi \mathrm{i} \operatorname{sg}(\operatorname{Im} \xi) \partial_{\xi} \Omega$.

Combining these equations we obtain

$$
\partial_{\xi} \Omega(\xi)=\frac{-\operatorname{sg}(\operatorname{Im} \xi)}{2 \pi \mathrm{i}} \int_{-\infty}^{\infty} \mathrm{d} u\left\langle m_{j} R_{0}\left(\rho_{j}-u\right) R_{0}(u)\right\rangle .
$$

In the same way we find from $(2.26 \mathrm{e})$, where $R_{2}(u)$ plays the role of $\partial_{\xi} E(u)$,

$$
\begin{aligned}
G(q, \xi)= & \frac{-\operatorname{sg}(\operatorname{Im} \xi)}{2 \pi \mathrm{i}} \int_{-\infty}^{\infty} \mathrm{d} u R_{0}(u)\left\langle\lambda_{j}^{*} R_{1}\left(\rho_{j}-u\right)+\lambda_{j} \bar{R}_{1}\left(\rho_{j}-u\right)\right. \\
& \left.+\lambda_{j}^{*} \lambda_{j} R_{0}\left(\rho_{j}-u\right)\right\rangle .
\end{aligned}
$$

Eq. (2.34) can also be proved from the definition ${ }^{15}$ )

$$
\partial_{\xi} \Omega^{(N)}=\frac{1}{N} \sum_{k=1}^{N}\left\langle m_{k} \mathscr{G}_{k k}^{(N)}\right\rangle=\frac{1}{N} \sum_{k=1}^{N}\left\langle\frac{m_{k}}{\rho_{k}-\frac{D_{k+2, N}}{D_{k+1, N}}-\frac{D_{1, k-2}}{D_{1, k-1}}}\right\rangle,
$$

where $\mathscr{G}_{k, 1}^{(N)}$ was defined in (2.11) and we used the equality

$$
D_{1, N}=\rho_{k} D_{1, k-1} D_{k+1, N}-D_{1, k-2} D_{k+1, N}-D_{1, k-1} D_{k+2, N} .
$$

For large $N$ the contributions from the terms with $1 \ll k \ll N$ will be equal and we thus have

$$
\partial_{\xi} \Omega=\left\langle\frac{m_{j}}{\rho_{j}-z-z^{\prime}}\right\rangle,
$$

where $z$ is the limit of $z_{N}$, defined in (2.20) and $z^{\prime}$ is a similar, but independent stochastic quantity; both are independent of $m_{j}$. This expression is equivalent to (2.34). In a similar manner eq. (2.35) can be deduced directly from definition (2.5a). In order to discuss the application of (2.34) to the density of states we put $\xi=-\omega^{2}+\mathrm{i} 0$ and introduce the "Schmidt"-function $W\left(u, \omega^{2}\right)$, which is the distribution function of $z_{N}\left(-\omega^{2}\right)$ as $\left.N \rightarrow \infty^{17,2}\right)$ :

$$
\begin{aligned}
W\left(u ; \omega^{2}\right) & =\lim _{N \rightarrow \infty}\left\{\operatorname{Prob}\left(z_{N}\left(-\omega^{2}\right)<u\right)-\operatorname{Prob}\left(z_{N}\left(-\omega^{2}\right)<0\right)\right\} \\
& =\theta(u)-\frac{1}{\pi} D\left(u+\mathrm{i} 0 ;-\omega^{2}+\mathrm{i} 0\right) .
\end{aligned}
$$


Existence of the limit and the second equality have been proved in $\left.{ }^{2}\right)$. It satisfies an equation, which follows from the equation (2.26a) for $D(u)$,

$$
W\left(u ; \omega^{2}\right)=\left\langle W\left(2-m_{j} \omega^{2}-\frac{1}{u} ; \omega^{2}\right)\right\rangle-W\left(-\infty ; \omega^{2}\right)-\theta(-u) .
$$

The integrated spectral density is given by

$$
H\left(\omega^{2}\right)=-W\left(-\infty ; \omega^{2}\right),
$$

which is seen from (2.24) and (2.39b). With this function the real and imaginary parts of (2.34) may be written for real $\omega$ as

$$
\begin{aligned}
& \frac{\mathrm{d}}{\mathrm{d} \omega^{2}} \gamma\left(\omega^{2}\right)=\text { P.V. } \iint_{-\infty}^{\infty} \mathrm{d} W\left(u ; \omega^{2}\right) \mathrm{d} W\left(u^{\prime} ; \omega^{2}\right) \int \mathrm{d} R\left(m_{j}\right) \frac{m_{j}}{u+u^{\prime}-2+m_{j} \omega^{2}}, \\
& \frac{\mathrm{d}}{\mathrm{d} \omega^{2}} H\left(\omega^{2}\right)=\int_{-\infty}^{\infty} \mathrm{d} W(u) \int \mathrm{d} R\left(m_{j}\right) m_{j} W^{\prime}\left(2-m_{j} \omega^{2}-u\right)
\end{aligned}
$$

where $R\left(m_{j}\right)$ is the common distribution function of the independently distributed masses $m_{j}(j=1,2,3, \ldots)$. An equation related to the last one was derived also by Halperin ${ }^{9}$; he also deduced an expression for the imaginary part of $G\left(q,-\omega^{2}-\mathrm{i} 0\right)$, related in a similar way to $(2.35)$.

The importance of eq. (2.42) lies in the fact that, contrary to the derivative of eq. (2.40), it relates $\mathrm{d} H / \mathrm{d} \omega^{2}$ to the behaviour of $W\left(u ; \omega^{2}\right)$ in its first variable $u$, which can be found from the solution of (2.39). Here we only focus on the question whether $\mathrm{d} H / \mathrm{d} \omega^{2}$ is a finite, continuous function of $\omega^{2}$; a similar analysis can be given for the imaginary part of $G\left(q,-\omega^{2}+\mathrm{i} 0\right)$. First, we assume that the mass distribution has a bounded density

$$
m_{j} R^{\prime}\left(m_{j}\right) \leqslant C .
$$

Extending the integral over $x_{j}=-m_{j} \omega^{2}$ to $(-\infty, \infty)$ and using $W(-\infty)-W(\infty)=1$ we obtain from (2.42) the bound

$$
\frac{\mathrm{d} H\left(\omega^{2}\right)}{\mathrm{d} \omega^{2}} \leqslant \frac{C}{\omega^{2}},
$$

which proves that it is finite, except possibly for $\omega \downarrow 0$ (there it behaves like $\left.\sqrt{\left\langle m_{j}\right\rangle} / 2 \omega\right)$. It is strictly positive, since else $W^{\prime}(u)$ has to vanish on a whole interval, which is also inconsistent with (2.39). From the continuity of $W\left(u, \omega^{2}\right)$ 
in $u$ (proven by Schmidt $\left.{ }^{17}\right)$ ) and in $\omega^{2} \dagger$ follows, again via (2.42), continuity of $\mathrm{d} H / \mathrm{d} \omega^{2}$. For the case that $R^{\prime}(m)$ has a power singularity, with power strictly less than one, it can be shown that the assumption that $W^{\prime}(u)$ has a power-or logarithmic singularity at some point $u_{0}$, is inconsistent with the convolutional structure of (2.39). This structure also causes $W$ to be absolutely continuous if $R(m)$ is. Therefore, it seems that for all absolutely continuous mass distributions the spectral density $\mathrm{d} H / \mathrm{d} \omega$ is a finite, continuous function of the frequency $\omega$. This conclusion was also reached by Wegner ${ }^{18}$ ) for tight binding electron models for distributions satisfying a similar bound as (2.43). For binary distributions of the masses, on the other hand, Schmidt ${ }^{17}$ ) showed that under some circumstances the function $W^{\prime}(u)$ is either zero or infinite on a set of points that is dense everywhere. If $W^{\prime}(u)$ is infinite on such a set, eq. (2.42) will probably yield an infinite value for $\mathrm{d} H / \mathrm{d} \omega^{2}$, i.e. the density of states does not exist for a binary mass distribution (at least not for high frequencies under some constraints on the values of the masses and the probabilities for their occurrence). This conclusion was reached, though not proved, by many authors: numerical evidence was found by Gubernatis and Taylor $^{19}$ ).

\section{Exact solutions for characteristic function and Green's function}

\subsection{Exponential distributions}

In this section we construct an explicit solution for the characteristic function and for the Green's function $\mathscr{G}=1 /(M \xi+\Phi)$. The starting point is (2.26) with $\lambda_{j}=1$, implying $\bar{R}_{1}(u ; q ; \xi)=R_{1}(u ;-q ; \xi)$. This set of equations will be solved exactly for the special case of exponential mass distributions. Hereto we put

$$
m_{j}=m+a x_{j}, \quad 0 \leqslant x_{j}<\infty,
$$

where $x_{j}$ has an exponential distribution

$$
r\left(x_{j}\right)=\mathrm{e}^{-x_{j}} .
$$

In a previous paper ${ }^{7}$ ) we have considered a random alloy model where there is a similar equation for $D(u)$. The method of solution given there for the special case of exponentially distributed $\delta$-potential strengths can be copied here and

† Continuity of $W\left(u, \omega^{2}\right)$ in $\omega^{2}$ is proven as follows: consider an infinite sequence $\omega_{j}^{2}$ with limit $\omega^{2}$. The sequence of non-decreasing functions $W\left(u, \omega_{j}^{2}\right)$ will have at least one convergent subsequence. If there are two of such subsequences, we define $\Delta W(u)$ as the difference of the two limit functions; this function satisfies the homogeneous version of (2.39), because $W$ is continuous in $u$. Then it is seen that $\Delta W(-\infty)=0$, because otherwise either the maximum or the minimum of $\Delta W(u)$ cannot be taken. The proof that $W=0$ for all $u$ goes analogous to the proof given in appendix B of ref. 2; the parameter $p$, occurring there, is equal to one. 
extended for the solution of the functions $R_{0}, R_{1}$ and $R_{2}$. The reason for solvability for the special choice of mass distributions (3.1) is the identity

$$
\left[1-a \xi u^{2} \partial_{u}\right] \int_{0}^{\infty} \mathrm{e}^{-x_{j}} \mathrm{~d} x_{j} D\left(2+m \xi+a \xi x_{j}-\frac{1}{u}\right)=D\left(2+m \xi-\frac{1}{u}\right),
$$

which is also valid for the functions $R_{0}, R_{1}$ and $R_{2}$. Therefore, eqs. (2.26) can be put in the form of differential-difference equations instead of integral equations,

$$
\left[1-a \xi u^{2} \partial_{u}\right] D(u)=D\left(2+m \xi-\frac{1}{u}\right)+\log \left(2+m \xi-\frac{1}{u}\right)-D(\infty)
$$

We define, with $\operatorname{Re} \mu \geqslant 0$,

$$
\cosh \mu=1+\frac{1}{2} m \xi, \quad \eta=\frac{a \xi}{2 \sinh \mu}
$$

and change variables by

$$
u=\frac{\mathrm{e}^{z}-1}{\mathrm{e}^{z} \cdot \mu}-\mathrm{e}^{\mu} ; \quad D(u(z))=G(z)+\log \frac{2 \sinh \mu}{1-\mathrm{e}^{-z}} .
$$

The resulting equation for $G(z)$ is a differential-difference equation

$$
\left[1+\eta\left(\mathrm{e}^{2}-2+\mathrm{e}^{-z}\right) \partial_{z}\right] G(z)=G(z+2 \mu)-\Omega+\mu+\eta\left(1-\mathrm{e}^{-z}\right),
$$

where we used the equality $D(\infty ; \xi)=\Omega(\xi)$. The solution is given by $\left.{ }^{7}\right)$

$$
G(z)=\sum_{k=1}^{\infty} c_{k} \frac{1-\mathrm{e}^{-k z}}{k}
$$

with $c_{k} \rightarrow 0$ as $k \rightarrow \infty$. The convergence of this expression for $\operatorname{Re}(z)>0$ is required, at least for $\xi$ real and positive, by arguments of analyticity, since $G(z)$ should have no singularities there. The coefficients $c_{k}$ satisfy the equations

$$
\begin{aligned}
& c_{1}=1-\frac{1}{\eta}(\Omega-\mu), \\
& c_{k+1}+c_{k-1}=\left(2+\frac{1-\mathrm{e}^{-2 k \mu}}{\eta k}\right) c_{k} \equiv \rho_{k} c_{k} \quad(k=1,2, \ldots),
\end{aligned}
$$

where we defined $c_{0}=1$. These equations have the same form as the equations of motion (2.1), but here there are no random parameters. This is a special property of the exponential distributions. The solution for the $c_{k}$, which satisfies the correct boundary conditions, has the form ${ }^{7}$ )

$$
c_{k}=A_{1} A_{2} \ldots A_{k}
$$


where the $A_{k}$ are continued fractions

$$
A_{k} \equiv \frac{c_{k}}{c_{k-1}}=\frac{1}{\rho_{k}-} \frac{1}{\rho_{k+1}-} \frac{1}{\rho_{k+2}-} \cdots .
$$

In particular we obtain from (3.7a) for the characteristic function the explicit expression

$$
\Omega=\mu+\eta\left(1-A_{1}\right) .
$$

In figs. 1a, b we present plots of the spectral density $\rho(\omega)=\mathrm{d} H / \mathrm{d} \omega$ and the exponential growth rate of inverse localization length $\gamma\left(\omega^{2}\right)$ for several values of $m$. We normalize the average of $m_{j}$ to unity, implying $0 \leqslant m \leqslant 1$ and $a=1-m$. A calculation of the specific heat has been reported in ${ }^{8}$ ).

Next we extend this method of solution to the other equations. The function $R_{0}(u)$ equals $D^{\prime}(u)+1 / u$; it becomes in terms of the variable $z$

$$
R_{0}(u(z))=\frac{-\mathrm{e}^{-z}\left(\mathrm{e}^{z-\mu}-\mathrm{e}^{\mu}\right)^{2}}{2 \sinh \mu} \sum_{k=1}^{\infty} c_{k} \mathrm{e}^{-k z}+\frac{1-\mathrm{e}^{2 \mu-z}}{2 \sinh \mu} .
$$

Using identity (3.2) the equation for $R_{1}(u)$ takes the form

$$
\left[1-2 \eta \sinh \mu u^{2} \partial_{u}\right] u \mathrm{e}^{-\mathrm{i} q} R_{1}(u)=R_{1}\left(2 \cosh \mu-\frac{1}{u}\right)+R_{0}\left(2 \cosh \mu-\frac{1}{u}\right) .
$$


Fig. 1. Spectral density $\rho$ and exponential growth rate $\gamma$ as function of the frequency $\omega$. For $\omega \downarrow 0$ : $\rho \simeq 1 / \pi, \gamma \simeq(1 / 8)(1-m)^{2} \omega^{2} ; \rho=0$ for $\omega>\omega_{\max }=2 / \sqrt{m}$. Except if $m=M=1$ (ordered case) $\rho \sim \exp \left(-A\left(\omega_{\max }-\omega\right)^{-\alpha}\right)$ for $\omega \uparrow \omega_{\max }$, with $\alpha=1.1 \pm 0.1$ (see ref. 7 for a similar situation). In the ordered case $m=M=1$ one has $\gamma=0$ inside the spectrum, because the eigenfunctions (plane waves) are not localized; $\gamma \simeq \log \omega^{2}+\left\langle\log m_{j}\right\rangle$ for $\omega^{2} \rightarrow \infty$. 
Defining similarly to (3.10)

$$
R_{1}(u(z))=\frac{\mathrm{e}^{z-\mu}-\mathrm{e}^{\mu}}{2 \sinh \mu} \sum_{k=1}^{\infty} d_{k} \mathrm{e}^{-k z},
$$

we obtain the set of equations, for $k=1,2, \ldots$,

$$
\begin{aligned}
& -k d_{k+1}-(k-1) d_{k-1}+\left(2 k-1+\frac{1}{\eta}\left(1-\mathrm{e}^{\mu+\mathrm{i} q-2 k \mu}\right)\right) d_{k} \\
& =\frac{\mathrm{e}^{\mathrm{i} q}}{\eta}\left(c_{k-1} \mathrm{e}^{2 \mu}-c_{k}\right) \mathrm{e}^{-2 k \mu} .
\end{aligned}
$$

They take simpler forms in terms of the quantities

$$
\delta_{k}=d_{k} / a_{k},
$$

where $a_{1}=\sqrt{\pi / 2}$ and

$$
a_{k+1}=\frac{1}{k a_{k}}=\frac{(k-1) ! !}{k ! !}\left\langle\begin{array}{ll}
a_{1}, & k \text { even, } \\
\frac{1}{a_{1}}, & k \text { odd },
\end{array}\right.
$$

which go like $1 / \sqrt{k}$ for large $k$. The equations for the $\delta_{k}$ are

$$
\tilde{\rho}_{k} \delta_{k}-\delta_{k+1}-\delta_{k-1}=\sigma_{k},
$$

with $\delta_{0}=0$ and

$$
\begin{aligned}
& \tilde{\rho}_{k}=a_{k}^{2}\left(2 k-1+\frac{1}{\eta}\left(1-\mathrm{e}^{\mu+\mathrm{i} q-2 k \mu}\right)\right), \\
& \sigma_{k}=a_{k} \frac{\mathrm{e}^{\mathrm{i} q-2 k \mu}}{\eta}\left(c_{k-1} \mathrm{e}^{2 \mu}-c_{k}\right) .
\end{aligned}
$$

They can be solved because they have the same structure as eq. (2.11) for $\mathscr{G}_{k l}$. The result is

$$
\delta_{k}=\sum_{i=1}^{\infty} \tilde{G}_{k l} \sigma_{l},
$$

with

$$
\tilde{\mathscr{G}}_{k l}= \begin{cases}\tilde{D}_{1, l-1} \tilde{c}_{k}, & k \geqslant l, \\ \tilde{D}_{1, k-1} \tilde{c}_{l}, & k \leqslant l .\end{cases}
$$

The determinants $\tilde{D}_{i, j}$ are defined by (2.12b) with $\rho_{k} \rightarrow \tilde{\rho}_{k}$ and $\tilde{c}_{k}=\tilde{D}_{k+1, \infty} / \tilde{D}_{k, \infty}$ is given by (3.8) after the same replacement. The expressions for the coefficients $d_{k}$ and $\bar{\delta}_{k}$, related in a similar way to $\bar{R}_{1}(u)$, follow after the replacement $q \rightarrow-q$. 
Finally, the equation for $R_{2}(u)$, corresponding to (3.11), has the form

$$
\begin{aligned}
& \left(1-2 \eta \sinh \mu u^{2} \partial_{u}\right) R_{2}(u)=R_{2}\left(2 \cosh \mu-\frac{1}{u}\right)+R_{1}\left(2 \cosh \mu-\frac{1}{u}\right) \\
& +\bar{R}_{1}\left(2 \cosh \mu-\frac{1}{u}\right)+R_{0}\left(2 \cosh \mu-\frac{1}{u}\right)+R_{2}(0) .
\end{aligned}
$$

Defining

$$
R_{2}(u(z))=R_{2}(0)+\sum_{k=1}^{\infty} e_{k} \frac{1-\mathrm{e}^{-k z}}{2 k \sinh \mu},
$$

we obtain for $e_{k}$ the equations

$$
\begin{aligned}
& \rho_{k} e_{k}-e_{k+1}-e_{k-1}=\frac{-1}{\eta} \tau_{k} \quad(k=1,2,3, \ldots), \\
& -R_{2}(0)=\frac{1}{2 \sinh \mu}\left(\mathrm{e}^{-\mu}\left(d_{1}+d_{1}\right)+1-c_{1} \mathrm{e}^{-2 \mu}-\eta e_{1}\right),
\end{aligned}
$$

where $\rho_{k}$ is defined in (3.7b), $e_{0} \equiv 0$ and

$$
\begin{aligned}
\tau_{k}= & \mathrm{e}^{\mu-2 k \mu}\left(-d_{k}-d_{k}+\mathrm{e}^{-2 \mu}\left(d_{k+1}+d_{k+1}\right)\right) \\
& +\mathrm{e}^{-2 k \mu}\left(-c_{k+1} \mathrm{e}^{-2 \mu}+2 c_{k}-c_{k-1} \mathrm{e}^{2 \mu}\right) \quad(k=1,2, \ldots) .
\end{aligned}
$$

We further define

$$
\tau_{0}=\mathrm{e}^{-\mu}\left(d_{1}+d_{1}\right)-c_{1} \mathrm{e}^{-2 \mu}+2 .
$$

The solution for (3.20a) follows again in the form of a Green's function, like in (3.16). In particular we obtain $e_{1}=(-1 / \eta) \sum_{k=1}^{\infty} c_{k} \tau_{k}$, with $c_{k}$ given in (3.8). Inserting this into (3.20b) the explicit expression function has the form

$$
\mathscr{G}(q, \xi)=\frac{1}{2 \sinh \mu}\left(-1+\sum_{k=0}^{\infty} c_{k} \tau_{k}\right) \text {. }
$$

It also follows from eq. (2.35) if we put $u \rightarrow \rho_{j}-u$ and use the equation of motion (2.26b) for $R_{0}(u)$. Another, equivalent expression for $\mathscr{G}(q, \xi)$ is obtained from (2.35) if one does not make this substitution and uses the equations of motion for $R_{0}, R_{1}$ and $\bar{R}_{1}$ :

$$
\mathscr{G}(q, \xi)=\frac{1}{2 \sinh \mu}\left(1+\sum_{k=0}^{\infty} c_{k} \tilde{\tau}_{k}\right),
$$

where

$$
\begin{aligned}
& \tilde{\tau}_{k}=\mathrm{e}^{-2 k \mu}\left(\mathrm{e}^{-\mathrm{iq} q}\left(d_{k+1}-d_{k}\right)+\mathrm{e}^{\mathrm{i} q}\left(d_{k+1}-d_{k}\right)+c_{k+1}-2 c_{k}+c_{k-1}\right), \\
& \tilde{\tau}_{0}=\mathrm{e}^{-\mathrm{i} q} d_{1}+\mathrm{e}^{\mathrm{i} q} d_{1}-2+c_{1} .
\end{aligned}
$$


The equality between (3.22) and (3.23) may be shown with the equations for the coefficients $c_{k}$ and $d_{k}$, though the proof is not trivial. Eq. (3.23) can be used as a check on a numerical calculation of the Green's function $\mathscr{G}(q, \xi)$.

\subsection{Gamma distributions}

In this section we consider the mass distributions with density

$$
r\left(m_{j}\right)=\frac{n}{\Gamma(n)} \mathrm{e}^{-n m_{j}\left(n m_{j}\right)^{n-1} \quad\left(0 \leqslant m_{j}<\infty\right) .}
$$

The average mass and width are given by

$$
\left\langle m_{l}\right\rangle=1 ; \quad \sqrt{\left\langle m_{j}^{2}\right\rangle-\left\langle m_{j}\right\rangle^{2}}=\frac{1}{\sqrt{n}} .
$$

For $n \rightarrow \infty$ the ordered case $\left(r\left(m_{j}\right)=\delta\left(m_{j}-1\right)\right)$ is recovered. The parameter $n$, occurring in (3.24) has to be positive; we will take it to be integer: $n=1,2,3, \ldots$. Performing $n$ partial integrations we obtain from (2.26a)

$$
\left(1-\frac{\xi}{n} u^{2} \partial_{u}\right)^{n} D(u)=D\left(2-\frac{1}{u}\right)+\log \left(2-\frac{1}{u}\right)-D(\infty)
$$

This equation is of "type $n$ " as defined in ${ }^{7}$ ), but here we are dealing with a parabolic Möbius transformation $u \rightarrow 2-1 / u$. The transformations used in section (3.1) are now singular. We define $D(u)=E(u /(u-1))$ which immediately yields a differential-difference equation

$$
\left(1+\frac{\xi}{n} v^{2} \partial_{v}\right)^{n} E(v)=E(v+1)+\log \frac{v+1}{v}-E(1)
$$

Writing $E$ as the Laplace transform of another function $G(z) / z$,

$$
E(v)=\int_{0}^{\infty} \frac{1-\mathrm{e}^{-z v}}{z} G(z) \mathrm{d} z-\log v,
$$

we obtain an ordinary differential equation for $G$,

$$
\left(1-\frac{\xi}{n} z \partial_{z}^{2}\right)^{n} G(z)=\mathrm{e}^{-z} G(z)
$$

and

$$
\Omega=E(1)=\sum_{k=1}^{n}\left(\begin{array}{l}
n \\
k
\end{array}\right)(k-1) !\left(\frac{-\xi}{n}\right)^{k} G^{(k)}(0) .
$$


The function $G$ is analytic at the origin and has to satisfy the boundary conditions

$$
G(0)=1 ; \quad G^{(k)}(z) \rightarrow 0 \quad \text { as } z \rightarrow \infty \quad(k=0,1, \ldots, 2 n-1) .
$$

Note that (3.30) is consistent with calculation of $E(1)$ from (3.28), using the equation (3.29) for $G(z)$. The mass distribution (3.24) with $n=1$ is a limiting case of the distribution of section 3.1. The equation for $G(z)$ can be obtained from the equations (3.7) for the coefficients $c_{k}$ in the limit $m \rightarrow 0, k \rightarrow \infty$ such that $z=2 k \sqrt{m \xi}$ remains fixed, if one identifies $G(z)=c_{k}$.

Eqs. (3.29) has been solved numerically for obtaining $\gamma\left(\omega^{2}\right)$ and $\rho(\omega)=\mathrm{d} H\left(\omega^{2}\right) / \mathrm{d} \omega$ for the case $n=1,2,3,4,5$; the results are presented in figs. 2a, b. This was facilitated by the fact that the asymptotic solutions $G_{1}, \ldots, G_{n}$ of (3.29) (with $\mathrm{e}^{-z}=0$ ) are proportional to modified Bessel functions;

$$
\begin{aligned}
& G_{1}(z)=2 \sqrt{\frac{n z}{\xi}} K_{1}\left(2 \sqrt{\frac{n z}{\xi}}\right), \\
& G_{l+1}(z)=z^{l+1} \partial_{z}^{l+2} G_{1}(z) \quad(l=1,2, \ldots, n-1) .
\end{aligned}
$$

For $\xi \rightarrow-\omega^{2}+\mathrm{i} 0$ the function $K_{1}$ changes into a Hankel function of the first kind. The solution was performed in three steps:

i) assume a Taylor expansion of $G(z)$ at $z=0$-this introduces $n$ free parameters - and integrate up to $z_{1}$;

ii) write for $z \gg 1$ (" $z=\infty$ ") as a linear combination of the asymptotic solutions (3.32) and integrate up to $z_{1}$;

iii) require that both values for $G(z)$ and its first $2 n-1$ derivatives match at $z=z_{1}$. These $2 n$ constraints fix the $n$ free parameters introduced at $z=0$ and the $n$ parameters introduced at $z=\infty$.
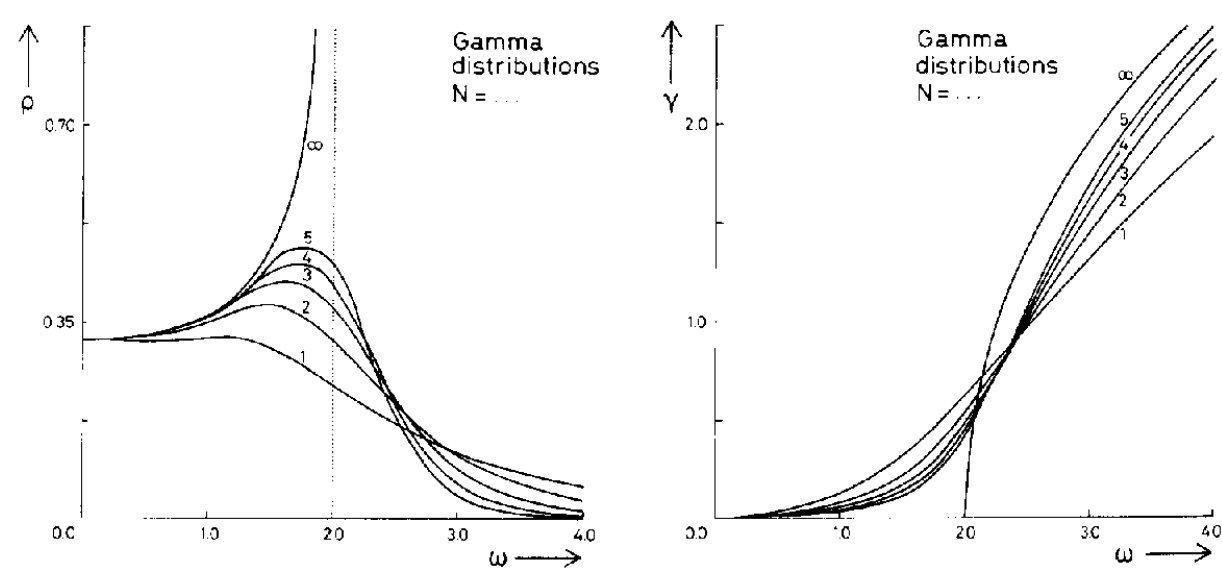

Fig. 2. Spectral density $\rho$ and exponential growth rate $\gamma$ as function of the frequency $\omega$. For $\omega \downarrow 0$ : $\rho \simeq 1 / \pi, \gamma \simeq(1 / 8 n) \omega^{2}$. For $\omega \rightarrow \infty, \rho \sim(2 n)^{n+1} \omega^{-2 n-1} / n !, \gamma \simeq \log \omega^{2}+\psi(n)-\log n$. 
The method becomes ineffective for small $\omega^{2}$ and large $n$, because the function $G(z)$ will oscillate rapidly; in these cases we could determine $\gamma$ and $H$ with enough accuracy from the Taylor expansion of the characteristic function $\Omega$ in powers of the frequency, as evaluated in ${ }^{8}$ ). From the definition ${ }^{2}$ )

$$
\Omega(\xi)=\left\langle\log \left(2+m_{1} \xi-\frac{1}{2+m_{2} \xi-}-\frac{1}{2+m_{2} \xi-} \cdots\right)\right\rangle
$$

one can show that for $\xi \rightarrow \infty: \Omega(\xi)=\log \xi\left(1-(-2 n / \xi)^{n} / n !+\cdots\right)+$ power series in $1 / \xi$. This yields the expression for the spectral density for large frequencies, as mentioned in the caption of fig. 2. From the results for the spectral density $\rho(\omega)$ the specific heat can be calculated; this has also been reported in ref. 8 . The equations for $R_{0}, R_{1}$ and $R_{2}$ which determine the Green's function $\mathscr{G}(q, \xi)$ also lead to ordinary inhomogeneous differential equations of order $2 n$, that can be solved easily for the simplest case $n=1$. We will not further elaborate on this, however.

\subsection{Expansion in cumulants}

In this section we introduce a scheme for a cumulant expansion of the Green's function, valid for arbitrary mass distributions. This expansion will turn out to be an expansion in powers of the frequency or, rather, in powers of $\sqrt{\xi}=\sqrt{-\omega^{2}}$. A related expansion for the characteristic function $\Omega(\xi)$ in powers of the variable $\sqrt{\langle m\rangle \xi /(1+\langle m\rangle \xi / 4)}$ was discussed in an earlier publication $\left.{ }^{8}\right)$, where the first nine Taylor coefficients were given explicitly. For the Green's function an expansion in cumulants is more appropriate. The starting point is eq. $(2.26 \mathrm{~b}-\mathrm{e})$. We introduce new variables and functions by

$$
\begin{aligned}
& \cosh \mu=1+\frac{1}{2}\langle m\rangle \xi, \\
& \begin{array}{l}
u=\frac{1-z}{\mathrm{e}^{\mu}-\frac{\mathrm{e}^{-\mu} z}{},} \\
R_{0}(u)=\frac{\mathrm{e}^{\mu}-\mathrm{e}^{-\mu} z}{2 \sinh \mu}\left\{\frac{-\mathrm{e}^{\mu}}{z}-\mathrm{e}^{-2 \mu}\left(\mathrm{e}^{\mu}-\mathrm{e}^{-\mu} z\right) T_{0}\left(z \mathrm{e}^{-2 \mu}\right)\right\}, \\
R_{1}(u)=\frac{\mathrm{e}^{\mu}-\mathrm{e}^{-\mu} z}{2 \sinh \mu\left(\mathrm{e}^{\mu-\mathrm{i} q}-1\right)}\left\{\frac{-\mathrm{e}^{\mu}}{z}+T_{1}\left(z \mathrm{e}^{-2 \mu}\right)\right\}, \\
\bar{R}_{1}(u)=\frac{\mathrm{e}^{\mu}-\mathrm{e}^{-\mu} z}{2 \sinh \mu\left(\mathrm{e}^{\mu+\mathrm{i} q}-1\right)}\left\{\frac{-\mathrm{e}^{\mu}}{z}+\bar{T}_{1}\left(z \mathrm{e}^{-2 \mu}\right)\right\}, \\
R_{2}(u)=\frac{1}{2 \sinh \mu\left(\mathrm{e}^{\mu-\mathrm{i} q}-1\right)\left(\mathrm{e}^{\mu+\mathrm{i} q}-1\right)}\left\{\frac{-\mathrm{e}^{\mu}\left(\mathrm{e}^{\mu}-\mathrm{e}^{-\mu} z\right)}{z}+T_{2}\left(z \mathrm{e}^{-2 \mu}\right)\right\} .
\end{array} .
\end{aligned}
$$


For the case $\lambda_{j}=1$ they satisfy the equations

$$
\begin{aligned}
\mathrm{e}^{-2 \mu} T_{0}\left(z \mathrm{e}^{-2 \mu}\right) & =\left\langle\frac{1}{(1+\beta \zeta)^{2}} T_{0}\left(\frac{z+\beta \zeta}{1+\beta \zeta}\right)+\frac{\beta}{z(z+\beta \zeta)}\right\rangle \\
T_{1}\left(z \mathrm{e}^{-2 \mu}\right)= & \left\langle\frac{\mathrm{e}^{\mu+\mathrm{i} q}}{1+\beta \zeta} T_{1}\left(\frac{z+\beta \zeta}{1+\beta \zeta}\right)+\frac{\mathrm{e}^{\mathrm{i} q} \beta \zeta}{z(z+\beta \zeta)}\right\rangle \\
& +\left(\mathrm{e}^{\mu}-\mathrm{e}^{\mathrm{i} q}\right)(z-1) \mathrm{e}^{-2 \mu} T_{0}\left(z \mathrm{e}^{-2 \mu}\right), \\
T_{2}\left(z \mathrm{e}^{-2 \mu}\right)= & \left\langle T_{2}\left(\frac{z+\beta \zeta}{1+\beta \zeta}\right)-\frac{\beta \zeta^{2}}{z(z+\beta \zeta)}\right\rangle+T_{2}\left(\mathrm{e}^{-2 \mu}\right) \\
& +(1-z)\left(\mathrm{e}^{\mu}-\mathrm{e}^{-\mathrm{i} q}\right) T_{1}\left(z \mathrm{e}^{-2 \mu}\right)+(1-z)\left(\mathrm{e}^{\mu}-\mathrm{e}^{\mathrm{i} q}\right) \bar{T}_{1}\left(z \mathrm{e}^{-2 \mu}\right) \\
& +(z-1)^{2}\left(\mathrm{e}^{\mu+\mathrm{i} q}-1\right)\left(\mathrm{e}^{\mu-\mathrm{i} q}-1\right) \mathrm{e}^{-2 \mu} T_{0}\left(z \mathrm{e}^{-2 \mu}\right),
\end{aligned}
$$

where $\zeta \equiv z-1$ and the random quantity $\beta$ is equal to

$$
\beta=\left(m_{j}-\left\langle m_{j}\right\rangle\right) \sqrt{\frac{\xi}{4\langle m\rangle+\langle m\rangle^{2} \xi}} .
$$

The equation for $\bar{T}_{1}$ is similar to that for $T_{1}$, with, however, $q \rightarrow-q$. The solutions to these equations are equal to zero if $\beta=0$, i.e. if there is no randomness. They can be solved sequentially in (averages of) powers of $\beta$. Before doing so we give the expression for eq. (2.35) in terms of the functions $T_{0}, T_{1}$ and $\bar{T}_{1}$ :

$$
\begin{aligned}
\mathscr{G}(q, \xi)= & \oint \frac{\mathrm{d} z}{2 \pi \mathrm{i} z}\left\{1+(z-1) \frac{1}{z^{2}} T_{0}\left(\frac{1}{z}\right)\right\}\left\{\mathscr{G}^{0}(q, \xi)-\frac{z T_{1}\left(z \mathrm{e}^{-2 \mu}\right)}{2 \sinh \mu\left(\mathrm{e}^{\mu}-\mathrm{e}^{\mathrm{i} q}\right)}\right. \\
& \left.-\frac{z \bar{T}_{1}\left(z \mathrm{e}^{-2 \mu}\right)}{2 \sinh \mu\left(\mathrm{e}^{\mu}-\mathrm{e}^{-\mathrm{i} q}\right)}+\frac{z(z-1) \mathrm{e}^{-2 \mu}}{2 \sinh \mu} T_{0}\left(z \mathrm{e}^{-2 \mu}\right)\right\},
\end{aligned}
$$

where $\mathscr{G}^{0}(q, \xi)$ is the Green's function in the ordered model with $m_{j}=\langle m\rangle$ :

$$
\mathscr{G}^{0}(q, \xi)=\frac{1}{2 \cosh \mu-2 \cos q}=\frac{1}{2+\langle m\rangle \xi-2 \cos q} .
$$

In order to explain the method we calculate $\mathscr{G}(q, \xi)$ up to fourth order in $\beta$.

We expand

$$
T_{0}(z)=T_{0}^{(2)}(z)+T_{0}^{(3)}(z)+T_{0}^{(4)}(z)+\cdots,
$$

where the superscripts indicate the order of $\beta$. Inserting this into (2.34a) and also expanding the argument of $T_{0}$ in the r.h.s., we obtain 


$$
\begin{aligned}
& T_{0}^{(2)}(z)=\frac{-\beta_{2}}{\left(\mathrm{e}^{2 \mu}-1\right) z^{2}}+\frac{\beta_{2}}{\left(\mathrm{e}^{4 \mu}-1\right) z^{2}}, \\
& T_{0}^{(3)}(z)=\frac{\beta_{3}}{\left(\mathrm{e}^{2 \mu}-1\right) z^{2}}+\mathcal{O}\left(\frac{1}{z^{3}}\right), \\
& T_{0}^{(4)}(z)=-3 \beta_{2}^{2}\left(\frac{1}{\mathrm{e}^{2 \mu}-1}+\frac{1}{\mathrm{e}^{4 \mu}-1}\right) \frac{1}{z^{2}}-\frac{\beta_{4}}{\left(\mathrm{e}^{2 \mu}-1\right) z^{2}}+\mathcal{O}\left(\frac{1}{z^{3}}\right),
\end{aligned}
$$

where $\beta_{k} \equiv\left\langle\beta^{k}\right\rangle$. Making a similar expansion for $T_{1}$ and using these results we find

$$
\begin{aligned}
& T_{1}^{(2)}(z)=\beta_{2}\left(\frac{A}{z}+\frac{B}{z^{2}}+\frac{C}{z^{3}}\right), \\
& T_{1}^{(3)}(z)=-\beta_{3} \frac{A}{z}+\mathcal{O}\left(\frac{1}{z^{2}}\right), \\
& T_{1}^{(4)}(z)=\frac{\mathrm{e}^{\mathrm{i} q}}{\mathrm{e}^{\mu}-\mathrm{e}^{\mathrm{i} q}} \beta_{2}^{2}(A-2 B+C) \frac{1}{z}+\beta_{4} \frac{A}{z}+\mathcal{O}\left(\frac{1}{z^{2}}\right),
\end{aligned}
$$

where

$$
\begin{aligned}
& A=\frac{-\mathrm{e}^{-2 \mu}}{2 \sinh \mu}-\frac{1}{\mathrm{e}^{\mu}-\mathrm{e}^{\mathrm{i} \psi}}, \\
& B=\frac{1}{\mathrm{e}^{4 \mu}-\mathrm{e}^{\mu+\mathrm{i} q}}\left[\left(\mathrm{e}^{\mu}-\mathrm{e}^{\mathrm{i} q}\right)\left(\frac{\mathrm{e}^{2 \mu}}{\mathrm{e}^{2 \mu}-1}+\frac{\mathrm{e}^{4 \mu}}{\mathrm{e}^{4 \mu}-1}\right)+2 \mathrm{e}^{\mathrm{i} q}\right], \\
& C=\frac{-\mathrm{e}^{-\mu}}{\mathrm{e}^{4 \mu}-1} .
\end{aligned}
$$

With these results the leading order terms of $\mathscr{G}(q, \xi)$ are found from (3.35)

$$
\begin{aligned}
\mathscr{G}(q, \xi)= & \mathscr{G}^{0}(q, \xi)+2 \sinh \mu \mathscr{G}^{0}(q, \xi)^{2}\left\{\beta_{2}-\beta_{3}+\left(\beta_{4}-3 \beta_{2}^{2}\right)\right\} \\
& +\beta_{2}^{2} \mathscr{G}^{0}(q, \xi)^{2}\left\{2 \cosh \mu+4 \sinh ^{2} \mu \mathscr{G}^{0}(q, \xi)\right. \\
& \left.+4 \sinh \mu \sinh 3 \mu \frac{\mathrm{e}^{\mathrm{i} q}}{\left(\mathrm{e}^{3 \mu}-\mathrm{e}^{\mathrm{i} q}\right)\left(\mathrm{e}^{\mathrm{i} q}-\mathrm{e}^{-3 \mu}\right)}\right\}+\mathscr{O}\left(\beta^{5}\right) .
\end{aligned}
$$

They determine long time tails of correlation functions in random relaxation models, see section 4.2. Higher order terms can be found in a similar fashion, but the method soon becomes cumbersome. This is related to the fact that for obtaining $\mathscr{G}$ three equations have to be solved sequentially.

In another approach Denteneer and Ernst $\left.{ }^{20}\right)$ start from the definition of $\mathscr{G}(q, \xi)$ and make an expansion in powers of the fluctuations $m_{j}-\left\langle m_{j}\right\rangle$. This leads them 
to multiple integrals of a special structure, which are performed analytically. In our method, however, there appear no integrals. Our equation (3.41) agrees with their results.

Finally, we mention that $\mathscr{G}(q, \xi)$ obeys a strange symmetry property: if we make the change $\mu \rightarrow-\mu$ (or $\sqrt{\xi} \rightarrow-\sqrt{\xi}$, or $\omega \rightarrow-\omega$ ) and replace the cumulants of the mass distribution $\left\langle\left\langle m^{k}\right\rangle\right\rangle$ by $(-1)^{k-1}\left\langle\left\langle m^{k}\right\rangle\right\rangle$, the Green's function remains invariant. In a previous publication $\left.{ }^{8}\right)$ we found that the characteristic function $\Omega$ - given by (2.25) as an integral over $\mathscr{G}$-changes sign under this transformation.

\section{Related models}

\subsection{Random masses and spring constants}

In this section we consider the problem of finding the characteristic function and Green's function for the more general equations of motion

$$
m_{j} \xi u_{j}=-L_{j} u_{j}+K_{j}\left(u_{j+1}-u_{j}\right)+K_{j-1}\left(u_{j-1}-u_{j}\right) .
$$

Here $K_{j}$ is the strength of the spring between the $j+1$ 'st and $j$ th particle and $L_{j}$ the strength of the spring between the $j$ th particle and its equilibrium position. In the model with $L_{j}=0$ Stephen and Kariotis ${ }^{16}$ ), using the replica method, have obtained the leading behaviour of $\gamma$ and $H$ (and thus of $\Omega$ ) as $\omega^{2} \rightarrow 0$, for several classes of distributions of $m_{j}$ and $K_{j}$.

In the case with constant $K_{j}(=1)$ the equations of section 2.1 are valid with $2+m_{j} \xi+L_{j}$ replacing $2+m_{j} \xi$. In particular, if $m_{j}=$ constant $=m$ and $L_{j}=L+a x_{j}$ with $r\left(x_{j}\right)=\mathrm{e}^{-x_{j}}$, the exact solutions of section (3.1) also hold for this case, with $\cosh \mu=1+\frac{1}{2} m \xi+\frac{1}{2} L$ and $\eta=a / 2 \sinh \mu$. If $m_{j}$ is distributed like in 3.1 and $L_{j}$ is constant the solution can also be extended, replacing $\cosh \mu$ by the expression mentioned above and leaving $\eta$ unchanged. If both $m_{j}$ and $L_{j}$ are random and have an exponential distribution, the model is of type $2^{7}$ ) and the equations for $\Omega(\xi)$ and $\mathscr{G}(q, \xi)$ can be cast in the form of 4-step recursion relations for the coefficients $c_{k}, d_{k}, \bar{d}_{k}$ and $e_{k}$.

The method for deriving the equations for the characteristic function and Green's function are similar to those of section 2.1; here we will only derive the equation for $D(u)$ and state without proof the resulting equations for the other functions. We define the quantity

$$
y_{j}=K_{j}\left(\frac{u_{j}}{u_{j+1}}-1\right),
$$

which satisfies

$$
y_{j}=-K_{j}+\frac{K_{j}^{2}}{K_{j}+\sigma_{j}-y_{j-1}},
$$


where

$$
\sigma_{j}=L_{j}+m_{j} \xi
$$

The function $D^{(N)}(u)$ will be defined by

$$
D^{(N)}(u)=\left\langle\log \left(y_{N}-u\right)\right\rangle \text {. }
$$

The limit function satisfies

$$
D(u)=\left\langle D\left(\sigma_{j}+\frac{u K_{j}}{u+K_{j}}\right)+\log \left(1+\frac{u}{K_{j}}\right)\right\rangle-\Omega .
$$

Here we used the equalities

$$
\left\langle D^{(N-1)}\left(\sigma_{N}+K_{N}\right)\right\rangle=\left\langle\log \left(-K_{N} \frac{u_{N+1}}{u_{N}}\right)\right\rangle \underset{N \rightarrow \infty}{\longrightarrow}\left\langle\log \left(-K_{j}\right)\right\rangle+\Omega ;
$$

the first one follows from (4.1) and in the second we used an equality for $\Omega(\xi)$-defined by (2.23)-deduced in ${ }^{2}$ ). The functions $R_{0}, R_{1}, \bar{R}_{1}$ and $R_{2}$ are defined similarly as in (2.22), with the same definition for $U$ and $\vec{U}$, but $y_{N}$ replacing $z_{N}$. They obey the equations

$$
\begin{aligned}
& R_{0}(u)=\left\langle\frac{K_{j}^{2}}{\left(u+K_{j}\right)^{2}} R_{0}\left(u^{\prime}\right)+\frac{1}{u+K_{j}}\right\rangle, \\
& R_{1}(u)=\mathrm{e}^{\mathrm{i} q}\left\langle\frac{K_{j}}{u+K_{j}}\left(R_{1}\left(u^{\prime}\right)+\lambda_{j} R_{0}\left(u^{\prime}\right)\right)\right\rangle \\
& R_{2}(u)=\left\langle R_{2}\left(u^{\prime}\right)+\lambda_{j}^{*} R_{1}\left(u^{\prime}\right)+\lambda_{j} \bar{R}_{1}\left(u^{\prime}\right)+\lambda_{j}^{*} \lambda_{j} R_{0}\left(u^{\prime}\right)\right\rangle-G(q, \xi)
\end{aligned}
$$

there is a similar equation for $\bar{R}_{1}$ and

$$
u^{\prime}=\sigma_{j}+\frac{K_{j} u}{u+K_{j}} .
$$

The expressions for $\Omega^{\prime}(\xi)$ and $G(q, \xi)$, corresponding to (2.34), (2.35), take the forms

$$
\begin{aligned}
& \Omega^{\prime}(\xi)=\frac{-\operatorname{sg}(\operatorname{Im} \xi)}{2 \pi \mathrm{i}} \int_{-\infty}^{\infty} R_{0}(u)\left\langle m_{j} R_{0}\left(\sigma_{j}-u\right)\right\rangle \mathrm{d} u, \\
& G(q, \xi)=\frac{-\operatorname{sg}(\operatorname{Im} \xi)}{2 \pi \mathrm{i}} \int_{-\infty}^{\infty}\left\langle R_{0}\left(\sigma_{j}-u\right)\left\{\lambda_{j}^{*} R_{1}(u)+\lambda_{j} \bar{R}_{1}(u)+\lambda_{j}^{*} \lambda_{j} R_{0}(u)\right\}\right\rangle .
\end{aligned}
$$

Next we try to construct solutions of these equations for the case $\lambda_{y}=1$ and appropriate choices of the distributions of the random variables. Dyson ${ }^{3}$ ) already 
noticed that, if $L_{j}=0$, the case with equal masses $m_{j}=m$ and random spring constants $K_{j}$ is equivalent to that with equal springs $K_{j}^{\prime}=c / m$ and random masses $m_{j}^{\prime}=c / K_{j}$. In order to show this in some detail we note that if $1 / K_{j}$ has an exponential distribution,

$$
\frac{1}{K_{j}}=\frac{1}{K}+\frac{1}{k} x_{j} ; \quad r\left(x_{j}\right)=\mathrm{e}^{-x_{j}},
$$

the equation for $D$ can be cast in the form

$$
\left(1+\frac{1}{k} u^{2} \partial_{u}\right) D(u)=\left\langle D\left(L_{j}+m_{j} \xi+\frac{K u}{K+u}\right)\right\rangle+\log \left(1+\frac{u}{K}\right)+\frac{1}{k} u-\Omega .
$$

If both $m_{j}$ and $L_{j}$ are constant this equation has a similar structure as eq. (3.3) and there will exist explicit expressions for $\Omega$ and $\mathscr{G}$. Making the transformation $D(u) \rightarrow D(1 /(1-\sigma / u))+\log u$, where $\sigma=L+m \xi$, and comparing with (3.3) we see that $\mu$ and $\eta$ are now given by $\cosh \mu=1+\sigma / 2 k$ and $\eta=\sigma / 2 k \sinh \mu$. In the case $L=0$ and $K=1$ they are indeed the same if we identify $k$ with $m / a$. In case $K=\infty$ eq. (4.11) becomes simpler and we can define

$$
D(u)=\int_{0}^{\infty} \frac{1-\mathrm{e}^{-z u}}{z} E_{0}(z)+D(0) .
$$

This form is consistent with the analytic properties of $D(u)$ for the case $\xi>0$, because then $y_{j}<0$ and $D$ has no singularities in the half plane $\operatorname{Re} u>0$. It solves the equation provided $E$ satisfies the ordinary differential equation

$$
\begin{aligned}
& E_{0}^{\prime \prime}(z)=\frac{1-\left\langle\mathrm{e}^{-z \sigma_{j}}\right\rangle}{k z} E_{0}(z), \\
& E_{0}(0)=1, \quad E_{0}(z) \rightarrow 0, \quad \text { if } z \rightarrow \infty ;
\end{aligned}
$$

the characteristic function is found as

$$
\Omega(\xi)=-E_{0}^{\prime}(0) \text {. }
$$

Note that this equation has a similar form as (3.29), (3.30) if $m_{j}$ and $L_{j}$ are non-random, in agreement with the similarity between the random mass and the random spring case, mentioned above. We go one step further, put $L_{j}=0$ and take as distribution of the $m_{j}$

$$
r\left(m_{j}\right)=p b e^{-b m_{j}}+q b^{2} m_{j} \mathrm{e}^{-b m_{j}}+r \delta_{+}\left(m_{j}\right), \quad\left(0 \leqslant m_{j}<\infty\right),
$$

where $p, q$ and $r$ are non-negative numbers, satisfying $p+q+r=1$. The equation 
for $E_{0}$ takes the form

$$
E_{0}^{\prime \prime}(z)=\left[\frac{(p+q) k}{(z+b / \xi)}+\frac{q b k}{\xi(z+b / \xi)^{2}}\right] E_{0}(z) .
$$

A similar equation was encountered before ${ }^{7}$ ) in a liquid alloy model. The solution of this equation, which satisfies the appropriate boundary conditions, is proportional to a modified Bessel function ${ }^{21}$ )

$$
E_{0}(z)=\sqrt{\frac{b+z \xi}{b}} \frac{K_{v}(\sqrt{4 k(p+q)(z+b / \xi)})}{K_{v}(\sqrt{4 k(p+q) b / \xi})},
$$

where $v=\sqrt{1+4 g b / k} \xi$. The characteristic function $\Omega$ then follows from (4.13b). In particular for the case $p=1, q=r=0$, where both the masses of the inverse of the spring constants $K_{j}$ have exponential distributions $\dot{\dagger}$, it is given by, with $m \equiv 1 / b=\left\langle m_{j}\right\rangle$,

$$
\Omega(\xi)=\sqrt{\frac{m \xi}{k}} \frac{K_{0}(2 \sqrt{k / m \xi})}{K_{1}(2 \sqrt{k / m \xi})} .
$$

The expression for $\gamma$ and $H$ take the form

$$
\begin{aligned}
& \gamma\left(\omega^{2}\right)=\sqrt{\frac{m \omega^{2}}{k} \frac{J_{0}(z) J_{1}(z)+Y_{0}(z) Y_{1}(z)}{J_{1}^{2}(z)+Y_{1}^{2}(z)}}\left(z=2 \sqrt{\frac{k}{m \omega^{2}}}\right), \\
& H\left(\omega^{2}\right)=\frac{m \omega^{2}}{k \pi^{2}\left(J_{1}^{2}(z)+Y_{1}^{2}(z)\right)} .
\end{aligned}
$$

For $\omega \rightarrow 0$ they have the asymptotic behaviour

$$
\gamma\left(\omega^{2}\right)=\frac{m \omega^{2}}{4 k}, \quad H\left(\omega^{2}\right)=\frac{\omega}{\pi} \sqrt{\frac{m}{k}} .
$$

The equation for $H$ agrees with the general expression $(\omega / \pi) \sqrt{\left\langle m_{j} / K_{j}\right\rangle}$; the expression for $\gamma$ has a term $(1 / 8)\left(\left\langle\left\langle m_{j}^{2}\right\rangle\right\rangle /\left\langle m_{j}\right\rangle\right)\left\langle 1 / K_{j}\right\rangle \omega^{2}$ from the randomness of the masses $\left.{ }^{13}\right)$ and a similar contribution $(1 / 8)\left(\left\langle\left\langle K_{j}^{-2}\right\rangle\right\rangle /\left\langle K_{j}^{-1}\right\rangle\right)\left\langle m_{j}\right\rangle \omega^{2}$ from the randomness of the springs. This shows that the localization length of the eigenfunctions - a measure for which is given by $1 / \gamma$, see ${ }^{1}$ )-is decreased by introducing more randomness in the system.

If $K_{j}$ is distributed according to (4.10) with $K=\propto$, the equations for $R_{1}, \bar{R}_{1}$ and $R_{2}$ can be cast in the form of second order differential equations too. We define

\footnotetext{
† Note that the function $K_{1}(z)$ also showed up in section 3.2 as asymptotic solution of the differential equation in the case of exponentially distributed $m_{j}$ and fixed $K_{j}$.
} 


$$
R_{1}(u)=\int_{0}^{\infty} \mathrm{e}^{-z u} E_{1}(z) \mathrm{d} z, \quad \bar{R}_{1}(u)=\int_{0}^{\infty} \mathrm{e}^{-z u} \bar{E}_{1}(z) \mathrm{d} z, \quad R_{2}(u)=-\int_{0}^{\infty} \frac{\mathrm{e}^{-z u}}{z} E_{2}(z) \mathrm{d} z
$$

and obtain for $E_{1}$ and $E_{2}$, which are analytic at $z=0$,

$$
\begin{aligned}
& z E_{1}^{\prime \prime}(z)+E_{1}^{\prime}(z)=k\left\{1-\mathrm{e}^{\mathrm{i} q}\left\langle\mathrm{e}^{-z \sigma_{j}}\right\rangle\right\} E_{1}(z)-k \mathrm{e}^{\mathrm{i} q}\left\langle\mathrm{e}^{-z \sigma_{j}}\right\rangle E_{0}(z), \\
& E_{2}^{\prime \prime}(z)=k \frac{E_{2}(z)}{z}+k\left\langle\mathrm{e}^{-z \sigma_{j}}\right\rangle\left\{\frac{-1}{z} E_{2}(z)+E_{1}(z)+\bar{E}_{1}(z)+E_{0}(z)\right\} .
\end{aligned}
$$

The boundary conditions are $E_{2}(0)=0, E_{1}, E_{2} \rightarrow 0$ as $z \rightarrow \infty$. The Green's function is given by $\mathscr{G}(q, \xi)=-(1 / k) E_{2}^{\prime}(0)$. The problem is now reduced to solving ordinary differential equations. We could not find, however, the exact solution for $E_{1}(z)$ in the case where $E_{0}(z)$ was proportional to a modified Bessel function. A similar situation seems to occur ${ }^{9}$ ) in the problem of an electron in a Gaussian white-noise potential, where the solution corresponding to $E_{0}(z)$ is proportional to an Airy function.

\subsection{Random relaxation models}

Another application of the results of the preceding sections lies in models of hopping classical particles described by Master equations of the form

$$
\dot{P}_{k}^{\mathrm{b}}=w_{k}\left(P_{k+1}^{\mathrm{b}}-P_{k}^{\mathrm{b}}\right)+w_{k-1}\left(P_{k-1}^{\mathrm{b}}-P_{k}^{\mathrm{b}}\right),
$$

or

$$
\dot{P}_{k}^{\mathrm{j}}=w_{k+1} P_{k+1}^{\mathrm{j}}+w_{k-1} P_{k-1}^{\mathrm{j}}-2 w_{k} P_{k}^{\mathrm{j}} .
$$

In the first case - "random barrier model", denoted by the superscript " $\mathrm{b}$ " $-w_{k}$ is the random frequency for hopping across a barrier between site $k$ and $k+1$; in the second one - "random jump model", denoted by the superscript " $j$ " $-w_{k}$ is the frequency for jumping from site $k$ to $k \pm 1$. An extensive discussion of the barrier model was given in a review by Alexander et al. ${ }^{15}$ ). In a particular case this model was used for the explanation of the temperature dependence of the exponent of the low frequency conductivity in the one-dimensional superionic conductor hollandite ${ }^{22}$ ). A recent discussion of the transparent properties of both models (4.22) is given by Denteneer and Ernst ${ }^{20}$ ). Here we follow these authors and shortly rederive the formulas for the Green's functions.

The solution of (4.22) follows by introducing the conditional probability $P(j, t \mid l, 0)$ - which is a Green's function-i.e. the probability that the walker is at site $j$ at time $t$ if he/she started at site $l$ at $t=0$. It also satisfies (4.22) with initial condition $P(j, 0 \mid l, 0)=\delta_{j, l}$. The solution follows by Laplace transform with 
respect to time:

$$
\begin{aligned}
& P^{\mathrm{b}}(j \mid l ; z) \equiv \int_{0}^{\infty} \mathrm{e}^{-z t} P^{\mathrm{b}}(j, t \mid l, 0)=\left(\frac{1}{E-1} \frac{1}{z 1+\Phi N}(E-1)\right)_{j l}, \\
& P^{\mathrm{j}}(j \mid l ; z)=\left(\frac{1}{z 1+\Phi N}\right)_{j l},
\end{aligned}
$$

Here 1 is the unit matrix, $N$ the diagonal matrix with elements $N_{i j}=\delta_{i j} w_{j} ; E$ and $E^{-1}$ matrices with elements $E_{i j}=\delta_{i, j+1}$ and $E_{i j}^{-1}=\delta_{i, j-1}$, respectively; $\Phi=2.1-E-E^{-1}$ is similar to the force matrix in section 2 . The moment generating function is defined as

$$
F(q, t)=\left\langle\mathrm{e}^{-\mathrm{i} q(n(t)-n(0))}\right\rangle \equiv\left\langle\sum_{j l} P(j, t \mid l, 0) P_{l}(0) \mathrm{e}^{-\mathrm{i} q(j-l)}\right\rangle,
$$

where $n(t)$ is the (discrete) position of the particle at time $t$. For describing diffusion in a stationary ensemble the distribution $P_{j}(0)$ of the starting points of the random walks has to be chosen as the stationary solution of (4.22) (similarly as for the van Hove $G(r, t)$ function). Thus we have

$$
\begin{aligned}
& P_{k}^{\mathrm{b}}(0)=\frac{1}{N}, \\
& P_{k}^{\mathrm{j}}(0)=\frac{m_{k}}{\Sigma_{l=1}^{N} m_{l}} ; \quad\left(m_{l} \equiv \frac{1}{w_{l}}\right),
\end{aligned}
$$

we will see in a moment that the parameter $1 / w_{j}$ indeed plays the role of the mass $m_{j}$ in the problem of the harmonic chain. Using this result and defining $M=N^{-1}\left(M_{i j}=\delta_{i j} m_{j}=\delta_{i j}\left(1 / w_{j}\right)\right)$ we can express the Laplace transform $G(q, z)=\int_{0}^{\infty} \mathrm{e}^{-z t} F(q, t)$, as $N \rightarrow \infty$, in the Green's function $\mathscr{G}(q, z)=\langle 1 /(M z+\Phi)\rangle_{q q}$, known from the preceding sections:

$$
\begin{aligned}
G^{\mathrm{b}}(q, z) & =\frac{1}{z}(1-\Phi(q) \mathscr{G}(q, z)), \\
G^{\mathrm{j}}(q, z) & =\frac{1}{z}-\frac{\Phi(q)}{\langle m\rangle z^{2}}(1-\Phi(q) \mathscr{G}(q, z)),
\end{aligned}
$$

where $\Phi(q)=2(1-\cos q)$. From the knowledge of $G(q, z)$ one can obtain information about the non-equilibrium properties of the system, like correlation functions of the form $\left\langle(n(t)-n(0))^{2 k}\right\rangle$ and the frequency dependent hopping conductivity $\sigma(\omega)$. This is described by the theory of generalized hydrodyna$\left.\operatorname{mics}^{23}\right)$. The generalized diffusion coefficient $U(q, z)$ is defined as

$$
G(q, z)=\frac{1}{z+q^{2} U(q, z)},
$$


where

$$
U(q, z)=U_{0}(z)-q^{2} U_{2}(z)+q^{4} U_{4}(z)+\cdots .
$$

The functions $U_{0}(z)$ and $U_{2}(z)$ are equal to

$$
\begin{aligned}
& U_{0}(z)=\frac{z^{2}}{2}\left\langle n^{2}(z)\right\rangle, \\
& U_{2}(z)=\frac{z^{2}}{24}\left\langle n^{4}(z)\right\rangle-\frac{z^{3}}{4}\left\langle n^{2}(z)\right\rangle^{2},
\end{aligned}
$$

where $\left\langle n^{2 k}(z)\right\rangle$ is the Laplace transform of $\left\langle(n(t)-n(0))^{2 k}\right\rangle$. The function $U_{0}(z)$ is the Laplace transform of $\frac{1}{2}\left(\mathrm{~d}^{2} / \mathrm{d} t^{2}\right)\left\langle(n(t)-n(0))^{2}\right\rangle$, which is the analogon of the velocity autocorrelation $\varphi_{2}(t)=\langle v(t) v(0)\rangle$ in ordinary hydrodynamics; $U_{2}(z)$ is related to the Burnett function. The frequency dependent hopping conductivity $\sigma(\omega)$ is related to $U_{0}(z)$ by a generalized Einstein relation ${ }^{24}$ ):

$$
\sigma(\omega)=\frac{n e^{2}}{k T} U_{0}(\mathrm{i} \omega),
$$

where $e$ is the charge and $n$ the density of the charged particles and $k$ the Boltzmann constant and $T$ the absolute temperature. Furthermore, since our underlying lattice is discrete, we write (4.28) in the form

$$
G(q, z)=\frac{1}{z+\Phi(q) V(q, z)},
$$

where

$$
V(q, z)=V_{0}(z)-\Phi(q) V_{2}(z)+\Phi(q)^{2} V_{4}(z)+\cdots .
$$

This is equivalent to a generalized diffusion equation for $\left\langle P_{n}(t)\right\rangle$ of the form

$$
\partial_{i}\left\langle P_{n}(t)\right\rangle=D(t) \Delta\left\langle P_{n}(t)\right\rangle+B(t) \Delta^{2}\left\langle P_{n}(t)\right\rangle+\cdots,
$$

where $D(t)$ and $B(t)$ are the inverse Laplace transforms of $V_{0}(z)$ and $V_{2}(x)$, respectively and $\Delta=E+E^{-1}-2$ is the discrete Laplace operator. The function $V$ can be obtained from (4.27);

$$
V^{\mathrm{b}}(q, z)=\frac{z \mathscr{G}(q, z)}{1-\Phi(q) \mathscr{G}(q, z)}
$$

for the barrier model and

$$
V^{\mathrm{i}}(q, z)=\frac{z(1-\Phi(q) \mathscr{G}(q, z))}{\langle m\rangle z+\Phi^{2}(q) \mathscr{G}(q, z)-\Phi(q)}
$$

for the jump model. 
From this it follows

$$
\begin{aligned}
& V_{0}^{\mathrm{b}}(z)=z \mathscr{G}(0, z) ; \quad V_{2}^{\mathrm{b}}(z)=-z^{2}(0, z)-\frac{1}{2} z \hat{C}_{q}^{2} \mathscr{G}(0, z), \\
& V_{0}^{\mathrm{i}}(z)=D ; \quad V_{2}^{\mathrm{i}}(z)=D \mathscr{G}(0, z)-D^{2} \frac{1}{z},
\end{aligned}
$$

where $D \equiv 1 /\langle m\rangle=1 /\langle 1 / w\rangle$ is the diffusion constant in the lattice. The fact that in the random jump model the diffusion coefficients $U_{0}(z)=V_{0}(z)$ simply equals a constant, was found before by Haus et al. ${ }^{25}$ ).

From the results of section 3 it follows that all these quantities are known explicitly for the exactly solved models where the "masses" $m_{j}=1 / w_{j}$ have exponential distributions. For arbitrary distributions we can obtain the small $z$-behaviour (or long time behaviour) of the functions $U_{0}(z)$ and $U_{2}(z)$ from the results of section 3.3 ,

$$
\begin{aligned}
& U_{0}^{\mathrm{b}}(z)=D\left(1+\frac{1}{2} \kappa_{2} \sqrt{z / D}+\left(-\frac{1}{4} \kappa_{3}+\frac{11}{24} \kappa_{2}^{2}\right) z / D+\mathcal{O}\left(z^{3 / 2}\right)\right) \\
& U_{2}^{\mathrm{b}}(z)=D\left(\frac{1}{12}+\frac{1}{108} \kappa_{2}^{2}+\frac{1}{24} \kappa_{2} \sqrt{z / D}+\text { other terms of order } \sqrt{z}+\cdots\right), \\
& U_{0}^{\mathrm{j}}(z)=D \\
& U_{2}^{\mathrm{j}}(z)=D\left(\frac{1}{12}+\frac{1}{2} \kappa_{2} \sqrt{D / z}-\frac{1}{4} \kappa_{3}+\frac{11}{24} \kappa_{2}^{2} \mathcal{O}(\sqrt{z})\right)
\end{aligned}
$$

where $\kappa_{k}=\langle m\rangle^{-k}\left\langle\left\langle m^{k}\right\rangle\right\rangle$ is proportional to the $k$ th cumulant. From this one finds, using Tauberian theorems, long time tails for the correlation functions $\varphi_{k+2}(t)=\mathscr{L}^{-1}\left(U_{k}(z)\right)(t)$

$$
\begin{aligned}
\varphi_{2}^{\mathrm{b}}(t) & \sim \frac{-\kappa_{2}}{4 \sqrt{\pi}(D t)^{3 / 2}}, \\
\varphi_{4}^{\mathrm{b}}(t) & \sim \frac{-\kappa_{2}}{48 \sqrt{\pi}(D t)^{3 / 2}}, \\
\varphi_{2}^{\mathrm{j}}(t) & =D \delta_{+}(t), \\
\varphi_{4}^{\mathrm{j}}(t) & \sim \frac{\kappa_{2}}{2 \sqrt{D \pi t}} .
\end{aligned}
$$

The $t^{-3 / 2}$ tail of $\varphi_{2}^{b}$ agrees with long time tails $\sim t^{-(d / 2+1)}$ found for the velocity autocorrelation function in $d$-dimensional Lorentz models ${ }^{26}$ ). The fact that $\varphi_{2}$ has no tail at all in the random jump model is compensated by a large $t^{-1 / 2}$ tail of $\varphi_{4}^{\mathrm{j}}$.

Alley and Alder ${ }^{27}$ ) introduce a modified Burnett coefficient, given by $U_{2}(0)$. In 
these discrete models its role is played by $V_{2}(0)$. This function is equal to $(D / 108) \kappa_{2}^{2}$ in the random barrier model, but diverges in the random jump model. We further note that eq. (4.35b) also has other contributions of order $t^{-3 / 2}$, involving products of cumulants. These as well as $1 / t$-corrections to these results, have been discussed by Denteneer and Ernst ${ }^{20}$ ).

Finally we consider the probability $P_{0}(t)$ that, at time $t$, the particle is still at or back at its initial position. Its Laplace transform is equal to $\left.\hat{P}_{0}(z)=\int_{0}^{\infty} \mathrm{e}^{-z t} P_{0}(t) \mathrm{d} t=\int_{-\pi}^{\pi} G(q, z) / 2 \pi\right) \mathrm{d} q$, as follows from (4.24). For the random barrier model, where $G$ is given by $(4.27 \mathrm{a})$, we recover eq. $(2.25)$ for the derivative of the characteristic function, $\Omega^{\prime}(z)$, in the harmonic chain with random masses. This again illustrates the close connection between these models, which, however, describe very different physical systems. Since the first nine coefficients of the expansion of $\Omega(z)$ in powers of $\sqrt{z}$ are known ${ }^{8}$ ), we can compute the long time behaviour of $P_{0}(t)$

$$
P_{0}(t)=\frac{\frac{1}{2}}{(\pi D t)^{1 / 2}}+\frac{1-\kappa_{3}+(15 / 16) \kappa_{2}^{2}}{32 \sqrt{\pi}(D t)^{3 / 2}}+\mathcal{O}\left(t^{-5 / 2}\right) .
$$

For most distributions of the variables $m_{j}=1 / w_{j}$, in particular for symmetric ones, the term $-\kappa_{3}+(15 / 16) \kappa_{2}^{2}$ is positive. For gamma distributions $\left(m_{j}=m+a x_{j}\right.$, with $r\left(x_{j}\right)$ given by (3.24)) this term is negative, however. For the exponential distribution $r\left(m_{j}\right)=\mathrm{e}^{-m_{j}}$ the whole term of order $t^{-3 / 2}$ in (4.36) is negative. This shows that the particle diffuses relatively fast from its starting point, caused by the asymmetry in the distribution of the jump frequencies. A similar behaviour is found in the problem of harmonic chains with random masses ${ }^{8}$ ), where the derivative of the specific heat increases or decreases for small temperatures, according to the same condition.

\section{3. $X-Y$ spins and tight binding electron models}

The one-dimensional $X-Y$ model for $S=\frac{1}{2}$ spins is described by the Hamiltonian

$$
\mathscr{H}=\sum_{j=1}^{N}\left\{2 J_{j}\left(S_{j}^{x} S_{j-1}^{x}+S_{j}^{y} S_{j-1}^{y}\right)+h_{j} S_{j}^{z}\right\} \quad\left(J_{1} \equiv 0\right) .
$$

It was considered first by Ruijgrok and Rodriguez ${ }^{28}$ ). The model where the $J_{j}$ are random and $h_{j}$ fixed was studied by $S{ }^{2}$ th $\left.^{29}\right)$. He showed that as in the ordered case $^{30}$ ) the Hamiltonian can be transformed in the following one:

$$
\mathscr{H}=\frac{1}{2} \sum_{j=1}^{N} h_{j}+\sum_{j=1}^{N}\left\{J_{j}\left(c_{j}^{\dagger} c_{j-1}+c_{j-1}^{\dagger} c_{j}\right)-h_{j} c_{j}^{+} c_{j}\right\},
$$

where the $c_{j}$ and $c_{j}^{\dagger}$ are fermion creation and annihilation operators. The constant 
term yields a shift to the internal energy and may further be omitted. Thus the problem is reduced to finding the eigenvalues of the equations

$$
J_{j} u_{j-1}+J_{j+1} u_{j+1}-h_{j} u_{j}=E u_{j} .
$$

These equations also occur in tight binding electron models, where $J_{j}$ is the hopping energy and the parameter $-h_{j}$ the site energy, as was noticed by Lieb et al ${ }^{30}$ ). We define the characteristic function in a similar way as in the case where $J_{j}=1$, see ref. 2 ,

$$
\begin{aligned}
\Omega(E) & =\lim _{N \rightarrow \infty} \frac{1}{N} \log \operatorname{sg}\left(J_{2} J_{3} \ldots J_{N+1}\right) u_{N+1}(E) \\
& =\int \mathrm{d} H(x) \log (E-x)-\left\langle\log \left|J_{j}\right|\right\rangle,
\end{aligned}
$$

where $u_{N+1}(E)$ is the solution of (4.39) with starting values $u_{0}=0, u_{1}=1$ and $H(E)$ is the accumulated density of states. For real values of $E \Omega(E)$ has the decomposition ${ }^{2}$ )

$$
\Omega(E \pm \mathrm{i} 0)=\gamma(E) \pm \mathrm{i} \pi(1-H(E)),
$$

where $\gamma(E)$ is the exponential growth rate or inverse localization length. For arbitrary complex values of $E$ the Green's function is defined as $\mathscr{G}(q, E)=\langle 1 /(E-\mathscr{H})\rangle_{q q}$. It determines the density of states at given values of $q$ and (real) $E$ as $\rho(q, E)=(1 / \pi) \operatorname{Im} \mathscr{G}(q, E-\mathrm{i} 0)$; the total density of states equals $H^{\prime}(E)=\int_{-\pi}^{\pi}(\mathrm{d} q / 2 \pi) \rho(q, E)$. Following the lines of section 2.1 we derive a set of equations for $\Omega$ and $\mathscr{G}$. Hereto we introduce the quantities, with $u_{0}=0, u_{1}=1$,

$$
z_{j}(E)=\frac{u_{j}}{J_{j+1} u_{j+1}}, \quad U_{j}(q, E)=\sum_{k=1}^{j} \frac{u_{\mathrm{k}} \mathrm{e}^{\mathrm{i} q(j+1-k)}}{J_{j+1} u_{j+1}}, \quad \overline{U_{j}}(q, E)=U_{j}(-q, E)
$$

and define functions $D(u), R_{0}(u), R_{1}(u), \bar{R}_{1}(u)$ and $R_{2}(u)$ by eq. (2.22). They satisfy the equations

$$
\begin{aligned}
& D(u)=\left\langle D\left(u^{\prime}\right)+\log \left(E+h_{j}-\frac{1}{u}\right)\right\rangle-D(\infty), \\
& R_{0}(u)=\left\langle\frac{1}{u^{2} J_{j}^{2}} R_{0}\left(u^{\prime}\right)\right\rangle+\frac{1}{u}, \\
& R_{1}(u)=\frac{\mathrm{e}^{i q}}{u}\left\langle\frac{1}{J_{j}} R_{1}\left(u^{\prime}\right)+\frac{1}{J_{j}^{2}} R_{0}\left(u^{\prime}\right)\right\rangle, \\
& R_{2}(u)=\left\langle R_{2}\left(u^{\prime}\right)+\frac{1}{J_{j}} R_{1}\left(u^{\prime}\right)+\frac{1}{J_{j}} \bar{R}_{1}\left(u^{\prime}\right)+\frac{1}{J_{j}^{2}} R_{0}\left(u^{\prime}\right)\right\rangle+R_{2}(0),
\end{aligned}
$$


where $u^{\prime} \equiv\left(E+h_{j}-1 / u\right) / J_{j}^{2}$ and $\bar{R}_{1}(u ; q ; E)=R_{1}(u ;-q ; E)$. The characteristic function is given by

$$
\Omega(E)=D(\infty ; E)-\left\langle\log \left|J_{j}\right|\right\rangle
$$

similar to eq. (2.34) its derivative equal to

$$
\Omega^{\prime}(E)=\frac{-\operatorname{sg}(\operatorname{Im} E)}{2 \pi \mathrm{i}} \int_{-\infty}^{\infty} \mathrm{d} u R_{0}\left(\frac{1}{u}\right)\left\{R_{0}(u)-\frac{1}{u}\right\} .
$$

The expression for $\mathscr{G}$ in terms of $R_{0}$ and $R_{\mathrm{I}}$ has the form

$$
\mathscr{G}(q, E)=\frac{-\mathrm{sg}(\operatorname{Im} E)}{2 \pi \mathrm{i}} \int_{-\infty}^{\infty} \frac{\mathrm{d} u}{u} R_{0}\left(\frac{1}{u}\right)\left\{\mathrm{e}^{-\mathrm{i} q} R_{1}(u)+\mathrm{e}^{\mathrm{i} q} \bar{R}_{1}(u)-u R_{0}(u)+1\right\} .
$$

With the identity

$$
J_{N+1}\left(u_{N+1}^{\prime}(E) u_{N}(E)-u_{N+1}(E) u_{N}^{\prime}(E)\right)=\sum_{k=1}^{N} u_{k}^{2}(E),
$$

it can be shown, similarly to (2.15)-(2.18), from the definition of $R_{2}(u)$ that $\mathscr{G}$ is equal to

$$
\mathscr{G}(q, E)=-R_{2}(0, q, E) .
$$

The signs of the $J_{j}$ have no influence on the eigenvalues of the energy; this can be seen, for instance, from the equation for $D(u)$, where only the square of $J_{j}$ enters. The Green's function, however, does depend on them. If the $J_{j}$ have a distribution which is symmetric around $J_{j}=0$, the Green's function does not depend on $q: \mathscr{G}(q, E)=\Omega^{\prime}(E)$. This is seen immediately from the eq. for $R_{2}(u)$ (4.42), where the $q$-dependent factors $R_{1}$ and $\bar{R}_{1}$ vanish. It can also be found using (4.42), (4.45).

\subsubsection{Diagonal disorder: random magnetic fields}

First we consider the case of constant $J_{j}$ ( $\equiv 1$ ), in which case the equations have the same form as those of the harmonic chain with random masses (section 2.1), with $E+h_{j}$ replacing $2+m_{j} \xi$. Exact solutions, like those discussed in section 3.1, exist for exponential distributions of the $h_{j}\left(h_{j}=\tilde{H}+h x_{j}\right.$ with $\tilde{H}, h \gtrless 0$ and $\left.r\left(x_{j}\right)=\mathrm{e}^{-x_{j}}\left(0 \leqslant x_{j}<\infty\right)\right)$. The parameters $\mu(\operatorname{Re} \mu \geqslant 0)$ and $\eta$ are defined by $\cosh \mu=\frac{1}{2}(E+\tilde{H}), 2 \eta \sinh \mu=h$. The boundaries of the spectrum in the ordered case $h=0$ occur for $E=-\tilde{H}+2(-1)^{l}(l=0,1)$, implying $\mu=-l \pi \mathrm{i}$. The values of $\Omega$ at these energies can be calculated from (3.9) by expanding the factors $\mathrm{e}^{-2 k \mu}$ 
in powers of $\mu-l \pi \mathrm{i}$. The first three terms are given by

$$
\Omega\left(-\tilde{H}+2(-1)^{h}\right)=-l \pi \mathrm{i}+\sqrt{(-1)^{\prime} h}-\frac{1}{8}(-1)^{l} h \quad(l=0,1) .
$$

Thus this is an expansion in powers of $\sqrt{(-1)^{h}}$, similar to an expansion in powers of the frequency for the disordered harmonic chain ${ }^{8}$ ). In case $h>0$ (the other case is similar) the spectrum lies between $-\infty<E<-\tilde{H}+2$. Combining (4.40b) and (4.48) we see that, for small $h, H(-\tilde{H}-2)=(1 / \pi) \sqrt{h}, \gamma(-\tilde{H}-2)=h / 8$ and $H(-\tilde{H}+2)=1$ (as should be, because all eigenvalues of the energy have values $\leqslant-\tilde{\mathbf{H}}+2)$ and $\gamma(-\tilde{H}+2)=\sqrt{h}-h / 8$. Similar properties were found in a random alloy model ${ }^{7}$ ). In the expression for $\Omega$, eq. (3.9), the density of states shows up i) for $-2 \leqslant E+\tilde{H} \leqslant 2$ because $\mu$ is imaginary; ii) for $(E+\tilde{H}) \operatorname{sg}(h) \leqslant-2$ because the continued fraction does not converge for these values of $E$ : see ${ }^{7}$ ) for a similar case.

We note that if the $J_{j}$ are random too, and take values \pm 1 with probabilities $p$ and $1-p$, respectively, the solutions for $\Omega$ and $\mathscr{G}$ take similar forms; if they have power law distributions, such as described in the following section, and the $h_{j}$ exponential distributions, the model is of "type 2" and the equations for $c_{k}$ and $d_{k}$ become four-step recurrence relations ${ }^{7}$ ).

\subsubsection{Off-diagonal disorder: random exchange constants}

The model where the $h_{j}$ are constant and the $J_{j}$ are random was considered by $\left.\mathrm{Smith}^{29}\right)$. He noticed that in this model the density of states is equal to the one in a harmonic chain, introduced by Dyson, where the ratios of the spring constants and the masses, $\lambda_{j}$, are independent identically distributed random variables. In the $X-Y$ model their role is played by the parameters $J_{j}^{2}$. Dyson found exact solutions for the case of gamma distributions (eq. (3.24)) for the $\lambda_{j}$. Smith considered them in great detail in order to show that the phase transition in the ordered $X-Y$ model at $T=0$ and $h= \pm 2 J$ is smeared out by the randomness. The model also has other exact solutions. In order to show this we choose the density of the $J_{j}$ as

$$
r\left(J_{j}\right)=\left\{\begin{array}{l}
p \frac{1}{\alpha} J_{j}^{1 / \alpha-1}, \quad J_{j} \geqslant 0, \\
(1-p) \frac{1}{\alpha}\left|J_{j}\right|^{1 / \alpha-1}, \quad J_{j} \leqslant 0,
\end{array}\right.
$$

where $\alpha$ is positive and $p$ is fraction of positive $J_{j}^{\prime}$ 's $(0 \leqslant p \leqslant 1)$. The absolute values of $J_{j}$ range between $0 \leqslant\left|J_{j}\right| \leqslant 1$. In the limit $\alpha \downarrow 0$ the distribution reduces to $\delta$ functions at $J_{j}= \pm 1$. For the distributions (4.49) the equation for $D(u)$ can be cast in the form of a complicated differential equation (we absorb $h$ into $E$ ): 


$$
\left[1-2 \alpha u^{2}\left(E-\frac{1}{u}\right) \partial_{u}\right] D(u)=D\left(E-\frac{1}{u}\right)+\log \left(E-\frac{1}{u}\right)-2 \alpha-D(\infty)
$$

We introduce new variables, with $\operatorname{Re} \mu \geqslant 0$,

$$
\begin{aligned}
& \cosh \mu=\frac{E}{2}, \quad u=\frac{\mathrm{e}^{2-\mu}-1}{\mathrm{e}^{z-2 \mu}-\mathrm{e}^{\mu}}, \\
& \eta=\frac{\alpha}{\sinh \mu}, \quad D(u)=\log \frac{2 \sinh \mu}{1-\mathrm{e}^{-z+\mu}}+G(z),
\end{aligned}
$$

implying $G(\mu)=0$. This function is subject to the equation

$$
\left\{1+2 \eta(\cosh z-\cosh \mu) \partial_{z}\right\} G(z)=G(z+2 \mu)+\mu-\Omega+\eta\left(\cosh \mu-\mathrm{e}^{-z}\right),
$$

which is slightly more complicated than eq. (3.5), but can be solved in the same way. We substitute $G(z)=\sum_{k=1}^{\infty} c_{k}\left(\mathrm{e}^{k \mu}-\mathrm{e}^{-k z}\right) / k$ and obtain for the coefficients $c_{k}$ the equations

$$
\begin{aligned}
& c_{k+1}+c_{k-1}=\left(2 \cosh \mu+\frac{1-\mathrm{e}^{-2 k \mu}}{\eta k}\right) c_{k} \equiv \rho_{k} c_{k}, \\
& \Omega=\mu+\eta\left(\cosh \mu-c_{1}\right),
\end{aligned}
$$

where we again defined $c_{0}=1$. From the definition of $G$ it can be shown that $c_{k}$ must go like $\exp (-k \mu)$ for $k \rightarrow \infty$. The corresponding differential equation for $R_{1}(u)$ is given by

$$
\left[1-\frac{2 \alpha}{1-\alpha} u^{2}\left(E-\frac{1}{u}\right) \partial_{u}\right] u\left\{\mathrm{e}^{-\mathrm{i} q} R_{1}(u)-u R_{0}(u)+1\right\}=r R_{1}\left(E-\frac{1}{u}\right),
$$

where $r=(2 p-1) /(1-\alpha)=\left\langle\operatorname{sg}\left(J_{j}\right)\right\rangle /(1-\alpha)$. Substitution of a Taylor series of the form (3.12) for $R_{1}(u)$ would yield here three-step recurrence relations; we therefore define

$$
\mathrm{e}^{-\mathrm{i} q} R_{1}(u)-u R_{0}(u)+1=\frac{\mathrm{e}^{z-2 \mu}-\mathrm{e}^{\mu}}{2 \sinh \mu} \sum_{k=1}^{\infty} d_{k} \mathrm{e}^{-k z} .
$$

The equations for the coefficients $d_{k}$ are two step recurrence relations, with $\tilde{\eta} \equiv \alpha /(1-\alpha) \sinh \mu$,

$$
\begin{aligned}
& -k d_{k+1}-(k-1) d_{k-1}+\left\{k \mathrm{e}^{\mu}+(k-1) \mathrm{e}^{-\mu}+\frac{1}{\tilde{\eta}}\left(1-r \mathrm{e}^{\mathrm{i} q+\mu-2 k \mu}\right)\right\} d_{k} \\
& =\frac{r}{\tilde{\eta}} \mathrm{e}^{\mathrm{i} q+\mu-2 k \mu}\left(c_{k-1} \mathrm{e}^{\mu}-c_{k}\right) \quad(k=1,2, \ldots) .
\end{aligned}
$$


They are very close to eq. (3.13) and their solution also runs along the lines (3.14)-(3.17). If we insert the expressions for $R_{0}(u)=D^{\prime}(u)+1 / u$ and $R_{1}(u)$ into (4.45) we obtain for the Green's function the closed expression

$$
\mathscr{G}(q, E)=\frac{1}{2 \sinh \mu} \sum_{k=0}^{\infty} \mathrm{e}^{-4 k \mu}\left(c_{k} \mathrm{e}^{-\mu}-c_{k+1} \mathrm{e}^{-4 \mu}\right)\left(d_{k+1}+\bar{d}_{k+1}+c_{k+1}-\mathrm{e}^{\mu} c_{k}\right),
$$

where $d_{k}(q, E)=d_{k}(-q, E)$. The equivalent of this expression in the random harmonic chain is given by (3.23).

We conclude this section with some remarks:

- in case $\alpha=0$ and $J_{j}$ are equal to +1 or -1 with probabilities $p$ and $1-p$, respectively. The characteristic function and Green's function can be found simply from (4.42),

$$
\begin{aligned}
& \Omega(E)=\mu(E), \\
& \mathscr{G}(q, E)=\frac{\sinh (\mu-\log |r|)}{2 \sinh \mu(\cosh (\mu-\log |r|)-\operatorname{sg}(r) \cos q)},
\end{aligned}
$$

where $r=2 p-1=\left\langle J_{j}\right\rangle$. The expression for $\Omega$ is the same as in the ordered case (all $J_{j}$ equal), because the signs of $J_{j}$ have no influence on it. The expression for the average one-particle Green's function $\mathscr{G}(q, E)$ has a similar structure as in the ordered case $(|r|=1)$, but it has no singularities; they have vanished due to the randomness.

- an argument of Lloyd $\left.{ }^{6}\right)$ states that if the $J_{j}$ are constant and the $h_{j}$ have Cauchy distributions $\left(h_{j}=\delta z_{j}, \tilde{r}\left(z_{j}\right)=1 /\left(\pi\left(1+z_{j}^{2}\right)\right),-\infty<z_{j}<\infty\right)$, the solution for the Green's function - and also the characteristic function - can be obtained from the one in the case $\delta=0$. Hereto one has to make the simple replacement $E \rightarrow E+\mathrm{i} \delta \operatorname{sg}(\operatorname{Im} E)$. We have observed that the argument is valid for a related liquid alloy model ${ }^{7}$ ) as well for the case where the equivalents of $h_{j}$ and/or $J_{j}$ are randomly distributed already and the (independent) Cauchy randomness is added to the $h_{j}$; this property also holds for the systems discussed in this section. Thus the solutions discussed in section 4.3.1 and given in (4.53), (4.57), (4.58) can be generalized to include this Cauchy distributed part of the $h_{j}$; the only difference is the above mentioned replacement in the definition of the parameter $\mu$.

- this argument also holds for the exact solutions obtained by Dyson ${ }^{3}$ ) for $\Omega$ for the case of gamma distributed $J_{j}^{2}$ (eq. (3.24)). The expression for $\Omega$ was also discussed in ref. $2 \dagger$ and is given by 


$$
\Omega(E)=\frac{\int_{0}^{\infty} \frac{\mathrm{d} v}{v}\left(\frac{v}{1+v}\right)^{n} \mathrm{e}^{-n v \xi} \log \frac{1}{v}}{\int_{0}^{\infty} \frac{\mathrm{d} v}{v}\left(\frac{v}{1+v}\right)^{n} \mathrm{e}^{-n v \xi}}-\psi(n)+\log n,
$$

where now $\xi(E)=-(E+\mathrm{i} \delta \operatorname{sg}(\operatorname{Im} E))^{2}$ and $\psi(n)$ is the logarithmic derivative of the gamma function. In particular it is seen that the divergency of $H^{\prime}(E)$ at $E=0$ has vanished since $\xi(0)=\delta^{2}>0$. From the result in Dyson's paper we find for small $\delta: H^{\prime}(0) \sim 1 / \delta(\log \delta)^{2}$, which indeed is finite.

\section{Summary}

In this paper we considered the problem of calculating the average one-particle Green's function in random one-dimensional systems. We start with a chain of harmonic oscillators with random masses (section 2.1 ). The method results in a recursively soluble set of equations for certain functions $R_{0}, R_{1}, \bar{R}_{1}$ and $R_{2}$; the Green's function follows from $R_{2}$. The function $R_{0}$ is related to the derivative of a function $D$, which we introduced before ${ }^{2}$ ) for obtaining the characteristic function (the analytic continuation of the accumulated spectral density into the complex frequency plane). Our set of equations is the generalization for complex frequencies of similar equations, obtained by Halperin ${ }^{9}$ ). They were deduced for obtaining the spectral density for given frequency and wavenumber, which is proportional to the imaginary part of the Green's function. The relation with his work is discussed. Whereas in his approach some "miracles" seem to happen, we derive the equations in a straightforward manner from the definition of the Green's function. In section 2.2 it is shown that knowledge of the function $R_{2}$ is not needed, in fact, for calculating the Green's function. A related property was observed by Halperin. Further we give a simple argument for the boundedness of the spectral density for mass distributions which have bounded densities (they are also allowed to have some power divergencies). It is made clear where the problems arise for binary distributions, where it is believed that the density of states does not exist as a smooth, bounded function.

In section 3.1 we show that for the case of exponential mass distributions exact solutions can be found, both for the characteristic function and the more complicated Green's function. Hereto we extend a method developed before for random Kronig-Penney models ${ }^{7}$ ). First the integral equations for the relevant functions are reduced in a number of steps to two-step difference equations. These equations have a similar structure as the original equation of motion, but there appear no random variables. Consequently, they can be solved with known 
methods. In section 3.2 we consider gamma distributions of the masses with integer parameter $n$. Here the above mentioned difference equations become ordinary differential equations of order $2 n$. As for the model of section 3.1, numerical calculations are presented for the density of states and the exponential growth rate or inverse localization length $\gamma$. In section 3.3 we discuss a scheme for obtaining an expansion of the Green's function in cumulants of the mass distribution. The method only requires algebraic manipulations. For the related problem of calculating the low frequency expansion of the characteristic function ${ }^{8}$ ) we have used a computer program for performing the algebraic manipulations and obtained the first twelve Taylor coefficients. They were used for calculating the specific heat as function of temperature.

In section 4.1 we extend the method to the more complicated case, where also the spring constants are random. We also allow for springs, that couple the particles to their equilibrium position. Since the problem of random masses and fixed springs is equivalent to the case where the masses are fixed and the springs are random, we also find an explicit solution if the inverse spring constants have exponential distributions. If both the masses and the inverse spring constants assume all positive values with exponential (or slightly more general) distributions, the characteristic function can be expressed in terms of modified Bessel functions. The equations for the Green's function then take the form of second order differential equations.

In section 4.2 we consider two models for random walks on a line, where the particle can jump to neighbouring sites i) across barriers with random height; ii) with jump frequencies, which differ from site to site. This problem is closely related to the harmonic chain with random masses or springs. We discuss some implications of the results obtained in previous sections, e.g. for the long time tails of certain correlation functions.

In section 4.3 we finally consider $X-Y$ and tight binding electron models. The case of diagonal disorder (random magnetic fields) is equivalent to the harmonic chain with random masses. In case of off-diagonal disorder (random exchange constants $J_{j}$ ) new solutions for the characteristic function and Green's function are deduced for power law distributions of the $J_{j}$. If they only take the values \pm 1 the solution has a simple form. If they have distributions, which are symmetric around $J_{j}=0$, the Green's function does not depend on $q$. Finally, an argument of Lloyd ${ }^{6}$ ) is applied to extend these solutions to the case where the magnetic fields (also) have Cauchy distributed parts; this has also been discussed for the solutions found by Dyson ${ }^{3}$ ).

It can be proven easily that in the harmonic chain with random masses the Green's function remains invariant under the transformation $\omega \rightarrow-\omega$, $\left\langle\left\langle m^{k}\right\rangle\right\rangle \rightarrow(-1)^{k-1}\left\langle\left\langle m^{k}\right\rangle\right\rangle$.

We note that the equations occurring in the problem of random harmonic 
chains also show up in models for electric lines with random capacitors and resistors ${ }^{3,15}$ ) and low lying excitations in Heisenberg magnets ${ }^{15}$ ).

There are several other approaches to the calculation of the Green's function. Stephen and Kariotis ${ }^{16}$ ) use replica methods and obtain a set of integral equations, which are used for studying the low frequency behaviour of random diffusion models, of the type considered in section 4.2 . They verify scaling assumptions made by Alexander et al. $\left.{ }^{15}\right)$. The relation between the replica method and the work of Dyson ${ }^{3}$ ) has been discussed by $\operatorname{Lin}^{32}$ ). The functions $D(u)$ and $R_{0}(u)$ (eq. (2.22)) and the function $Q(V)$ considered by Stephen and Kariotis are different extensions of Dyson's functions (see ref. 2) and for a similar case ${ }^{32}$ ), respectively). In a similar way, eq. (4.5) of Stephen and Kariotis is related to our eqs. (2.26c, d). Because in their equations an additional integral occurs (related to the transformation $u \rightarrow$ const $-1 / u$ in (2.26)), their method seems to be more complicated than ours; it is not clear, e.g., how the exact solutions, discussed in this paper, can be obtained from their formalism. Tao and Luttinge ${ }^{31}$ ) consider random Kronig-Penney models and use a modified version of the replica method. They assume that the potential has zero range and reduce the problem to that of linear partial differential equations. After spatial Fourier transformation they become ordinary differential equations. These equations are equivalent to eq. (4.13), (4.21) of the harmonic chain with exponentially distributed inverse spring constants. The method of this paper can also be applied to these models and, more generally, to all Kronig-Penney models discussed in ref. 7 . There will also be exact solutions for the Green's function, for the model for which in that paper the characteristic function has been solved explicitly. The solution follows by solving a slightly more general form of the difference equations discussed in section 3.1. In limiting cases these difference equations become ordinary differential equations (see sections 3.2, 4.1) and it turns out that these are just the cases treated by Tao and Luttinger. Denteneer and Ernst $^{20}$ ) study transport properties of random hopping models and make a cumulant expansion for the Green's function, starting from its definition. This method, as well as the scheme discussed in section 3.3, is straightforward, but soon becomes cumbersome, because many different terms arise.

We have not considered the calculation of two-particle Green's functions. They were discussed by Halperin ${ }^{9}$ ) and Tao and Luttinger ${ }^{31}$ ). From our experience with one-particle Green's functions and the structure of their equations we expect that a similar scheme can be developed for functions in two variables and that for exponential distributions these equations can be cast in difference equations in two discrete variables. In limiting cases these will become partial differential equations. We conclude remarking that interesting physical properties, like diffusion of momentum in random harmonic chains, are determined by the Green's function and known, in principle, in the exactly solved models. 


\section{Acknowledgements}

It is a pleasure to thank Professors N.G. van Kampen, B.R.A. Nijboer and Th.W. Ruijgrok for discussions on general aspects of Green's functions; Professor M.H. Ernst and P.J.H. Denteneer for introducing me to the Green's function approach in the subject of random walks on random lattices and for communicating their results prior to publication; Dr. J. Groenveld for discussions on aspects of the Schmidt functions and finally Professor Th.W. Ruijgrok and Bela Mulder for critical remarks on the manuscript.

\section{References}

1) D.J. Thouless, J. Phys. C (Solid St. Phys.) 5 (1972) 77.

2) Th.M. Nieuwenhuizen, Physica 113A (1982) 173.

3) F.J. Dyson, Phys. Rev. 92 (1953) 1331.

4) C. Domb, A.A. Maradudin, E.W. Montrol and G.H. Weiss, Phys. Rev. 115 (1959) 24.

5) H.L. Frisch and S.P. Lloyd, Phys. Rev. 120 (1960) 1175.

7) Th.M. Nieuwenhuizen, J. Phys. A (1984).

8) Th.M. Nieuwenhuizen, Physica 120A (1983) 468.

8) T.M. Nieuwenhuizen, chapter III of this thesis.

9) B.I. Halperin, Phys. Rev. 139 (1965) A104.

10) I.U. Heilmann, J.D. Axe, J.M. Hastings, G. Shirane, A.J. Heeger and A.G. MacDiarmid, Phys. Rev. B20 (1979) 751 .

11) S. Doniach and E.H. Sondheimer, Green's Functions for Solid State Physicists (Benjamin, London, 1974).

12) D.C. Herbert and R. Jones, J. Phys. C (Solid St. Phys.) 4 (1974) 1145.

13) H. Matsuda and K. Ishii, Supp. Prog. Th. Ph. 45 (1970) 56.

14) R. Carmona, Duke Math. J. 49 (1982) 191.

15) S. Alexander, J. Bernasconi, W.R. Schneider and R. Orbach, Rev. Mod. Phys. 53 (1981) 175.

16) M.J. Stephen and R. Kariotis, Phys. Rev. B26 (1982) 2917.

17) H. Schmidt, Phys. Rev. 105 (1957) 425.

18) F.J. Wegner, Z. Phys. B44 (1981) 9.

19) J.E. Gubernatis and P.L. Taylor, J. Phys. C (Solid St. Phys.) 4 (1971) L94.

20) P.J.H. Denteneer and M.H. Ernst, J. Phys. C (Solid St. Phys.) 16 (1983) L961.

21) M. Abramowitz and I.A. Stegun, Handbook of Mathematic Functions (Dover, New York, 1972).

22) J. Bernasconi, H.U. Beyeler, S. Sträsler and S. Alexander, Phys. Rev. Lett. 42 (1979) 819.

23) I.M. de Schepper and M.H. Ernst, Physica 98A (1979) 189. M.H. Ernst and H. van Beyeren, J. Stat. Phys. 26 (1981) 1.

H. van Beyeren, Rev. Mod. Phys. 54 (1982) 1.

24) H. Scher and M. Lax, Phys. Rev. B7 (1973) 4491, 4502.

25) J. Haus, K. Kehr and J. Lyklema, Phys. Rev. B25 (1982) 2905.

26) M.H. Ernst and A. Weyland, Phys. Lett. 34A (1971) 39.

27) B.J. Alder and W.E. Alley, J. Stat. Phys. 19 (1978) 341.

28) T.W. Ruijgrok and S. Rodriguez, Phys. Rev. 119 (1959) 596.

29) E.R. Smith, J. Phys. C (Solid St. Phys.) 3 (1970) 1419.

30) E. Lieb, T. Schultz and D. Mattis, Ann. Phys. N.Y. 16 (1961) 407.

31) R. Tao and J.M. Luttinger, Phys. Rev. B27 (1983) 935.

32) T.F. Lin, Journ. of Math. Ph. 11 (1970) 1584. 